\title{
Aerodynamic Characteristics of Radar Antenna in Stationary and Azimuthal Rotational Motion
}

\author{
Yanqi Zhang $\mathbb{i}$ and Zhaoming Zhang \\ Key Lab of Ministry of Industry and Information Technology for Unsteady Aerodynamics and Flow Control, \\ College of Aerospace Engineering, Nanjing University of Aeronautics and Astronautics, Nanjing, Jiangsu 210016, China
}

Correspondence should be addressed to Yanqi Zhang; yanqizhang_nuaa@163.com

Received 23 July 2021; Accepted 12 November 2021; Published 30 November 2021

Academic Editor: Stefano Selleri

Copyright (c) 2021 Yanqi Zhang and Zhaoming Zhang. This is an open access article distributed under the Creative Commons Attribution License, which permits unrestricted use, distribution, and reproduction in any medium, provided the original work is properly cited.

\begin{abstract}
To study the effects of aerodynamic loads on the aerodynamic characteristics of stationary and azimuthally rotating antennas, wind tunnel force tests are conducted using solid and porous plate antennas. The variation of aerodynamic coefficient with azimuth angle is obtained when the antenna is stationary and azimuthal rotation, and the results are compared with those from numerical simulations. The variation in the aerodynamic coefficients with respect to the azimuth angle is found to be sinusoidal for both the solid and porous plate antennas rotating in azimuth. Compared with the antenna stationary, quantitative analysis indicates that the rotational motion increases the maximum value and root mean square of the aerodynamic coefficient. For solid plate antenna, $\left|C_{x}\right|_{\_\max },\left|C_{m y}\right|_{\_\max }$, and $\left|C_{m z}\right|_{\_\max }$ increase by $41.6 \%, 15.0 \%$, and $47.3 \%$, respectively; $C_{x \_ \text {rms }}, C_{m y \_r m s}$, and $C_{m z \_r m s}$ increase by $19.0 \%, 20.0 \%$, and $19.1 \%$, respectively. For porous plate antenna, $\left|C_{x}\right|_{\max },\left|C_{m y}\right|_{\max }$, and $\left|C_{m z}\right|_{\max }$ increase by $30.6 \%$, $71.4 \%$, and $40.9 \%$, respectively; $C_{x_{-} \text {rms }}, C_{m y \_r m s}$, and $C_{m z \_r m s}$ increase by $22.9 \%, 50 \%$, and $20 \%$, respectively. The wind tunnel tests verify the feasibility of using numerical simulations to obtain the flow field results. By analyzing the surface pressure coefficient and vortex core track distribution, the effects of azimuthal rotation on the aerodynamic characteristics of the antenna are further clarified.
\end{abstract}

\section{Introduction}

The aerodynamic load of large-scale rotating radar antennas is constantly changing. Thus, it is necessary to consider not only the aerodynamic characteristics of the antenna at rest but also the aerodynamic characteristics of rotating radar antennas in terms of the rigidity and strength of the antenna structure and the servo system [1]. For this purpose, the dynamic wind load must be considered in the design of radar antennas [2-4]. In the past, researchers have mainly focused on the aerodynamic characteristics of the antenna when it is stationary [5-7]. Lombardi [2] and Blaylock et al. [6] obtained the surface pressure distribution of parabolic antennas through wind tunnel pressure tests and studied the effects of the porosity rate, focal diameter ratio, ground clearance, rotation center, support structure, and other factors on the surface pressure distribution of the antennas.
Wyatt [8] and Martín et al. [9] used wind tunnel static force tests to study the aerodynamic characteristics of radar antennas with different porosity rates and ground clearances.

The difference in the aerodynamic characteristics of radar antennas under static and rotating conditions mainly depends on the antenna type and the rotation parameters [10]. Lombardi [3] studied the aerodynamic characteristics of a shipborne radar antenna during azimuthal rotation. Significant differences were found between the drag, lift, and azimuth moment coefficient when the antenna is stationary and the drag, lift, and azimuth moment coefficient when the antenna is rotating azimuthally. The limitations of the semiempirical formula for converting static aerodynamic data into dynamic aerodynamic data have been verified, illustrating the importance of wind tunnel dynamic force tests in studying of aerodynamic characteristics of radar antenna rotating in the azimuth [10]. 
In studies on the aerodynamic characteristics of radar antennas, numerical simulations can provide more flow field details than wind tunnel tests [11]. Du and Du [12] studied the wind pressure distribution on the radar antenna surface in the atmospheric boundary layer using a time history method to calculate the wind load on the antenna surface in the atmospheric boundary layer. Through numerical simulations of the surface wind pressure distribution of a phased radar antenna array, the effect of the atmospheric boundary flow conditions on the radar antenna surface wind pressure distribution was analyzed, and the model blocking rate and wind tunnel wall interference on the rectangular antenna revealed the effect of using numerically simulated aerodynamic coefficients. Besides, the numerical results were found to be in good agreement with wind tunnel test results [13].

Research on the aerodynamic characteristics of radar antennas using numerical simulations not only considers the surface pressure and aerodynamic coefficient. As the radar antenna rotates, the resulting vortex structure affects the aerodynamic characteristics of the antenna. Gumusel et al. [14] and Camci and Gumusel [15] used numerical simulations to determine the flow field distribution of an antenna. The fluid-structure interaction between the antenna and its shedding vortices was found to be the main reason for antenna noise and vibration. Most existing radar antennas have a mostly blunt body. The unsteady aerodynamic characteristics of bluff body models in pitch or azimuthal rotation have been studied by $\mathrm{Yu}$ et al. [16] and $\mathrm{Wu}$ et al. [17], who successfully captured the vortex structure of a plate in pitching motion using numerical methods. Their results show that the difference between the aerodynamic characteristics when the antenna is stationary and the aerodynamic characteristics when the antenna is rotating may be caused by significant differences in the shedding vortex structures. Hargreaves et al. [18] and Gu et al. [19] studied the rotation of the plate model around a fixed axis and found that the surface pressure distribution and the vortex structure around the plate change periodically.

In general, there are several problems to be solved regarding the aerodynamic characteristics of radar antennas in azimuthal rotation. In terms of wind tunnel tests, the limitations of the related dynamic force measurement test conditions mean that existing research contents focus on the aerodynamic characteristics of the antenna at rest, while the aerodynamic characteristics of antenna rotation have rarely been studied. Without knowing the difference between them, it is impossible to design a reasonable and effective antenna servo system or to investigate the wind resistance performance. Regarding numerical simulations, the shape, boundary conditions, and material properties need to be simplified, which will have a certain impact on the simulation results. Additionally, the existing numerical simulation results for radar antennas in azimuthal rotation lack the support of wind tunnel test data. As the semiempirical formula only applies to specific antenna shapes, it has significant limitations in practical engineering applications, and its accuracy needs to be verified by wind tunnel tests.

In this study, the aerodynamic characteristics of solid and porous plate antennas are obtained using a wind tunnel dynamic force test platform, and the quantitative differences of aerodynamic characteristics between the stationary and azimuthal rotation of antenna are analyzed. The variations in the antenna aerodynamic coefficients with azimuth angle are thus obtained. By comparing the wind tunnel test results with those from numerical simulations, the feasibility of using numerical methods to analyze the structure distribution of the surrounding pressure field and vorticity field when the antenna is stationary and rotating is verified. Comparing the differences in the flow fields then reveals the effect of the rotation on the aerodynamic characteristics of antennas.

\section{Experimental Methods}

2.1. Wind Tunnel and Test Model. Experiments were carried out in the NH-2 wind tunnel at the Laboratory of Aerodynamics at Nanjing University of Aeronautics and Astronautics. The NH-2 wind tunnel is a closed-circuit, lowspeed wind tunnel with a test section measuring $3 \mathrm{~m}$ (width) $\times 2.5 \mathrm{~m}$ (height) $\times 6 \mathrm{~m}$ (length). The wind tunnel has a $2.89: 1$ contraction ratio, a flow velocity range of $0-93 \mathrm{~m} / \mathrm{s}$, and a turbulence intensity range of $0.10-0.14 \%$.

The radar antenna model for the test has three parts: a plate antenna, rod, and elevation angle adjustment mechanism. Since the solid type and porous type are the two most commonly used plate antennas that can rotate in azimuth, two types of plate antenna models are considered in this study: a solid plate and a porous plate. The antenna models are shown in Figures 1(a) and 1(b), the definitions are shown in Figure 1(c), and the detailed parameters are presented in Table 1 . As the maximum blockage rate of the model is less than $2.5 \%$, according to Blaylock et al. [6], no blockage correction has been made.

2.2. Apparatus and Testing Procedure. The radar antenna is mounted on a six-component box strain balance, which is connected to the base below the wind tunnel test section by a DD motor, as indicated in Figure 2(a). Using amplifiers and a PCI-1742U high-resolution multifunction data acquisition card, the aerodynamic loads can be measured at frequencies up to $1 \mathrm{MHz}$. A PCI-1245 motion control card and a highresolution incremental encoder are used to achieve precise adjustment of the azimuth angle and the rotation speed of the DD motor. The model motion control and data acquisition system are shown in Figure 2(b).

The strain-gage balance provides six-component force data, which are converted into the load signal using the balance formula in the AIAA strain-gage standard [20]. Zero-point reference sets are obtained when there is no wind at each data point, and these are used to subtract gravity effects and the inertia force from the wind aerodynamic load of the model.

In the wind tunnel static force test, the sampling aerodynamic load is collected at a sampling frequency of $1 \mathrm{kHz}$ over a sample collection time of $4 \mathrm{~s}$. The data are then averaged to give the mean aerodynamic force. The test is carried out in a uniform flow of $25 \mathrm{~m} / \mathrm{s}$ for the porous plate 


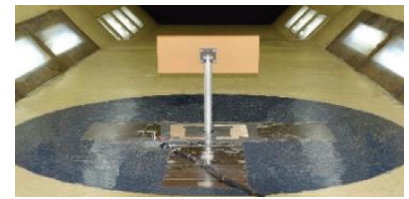

(a)

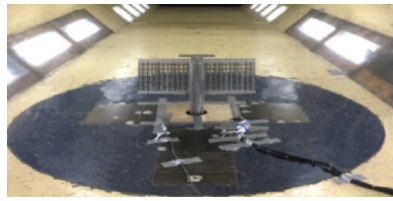

(b)

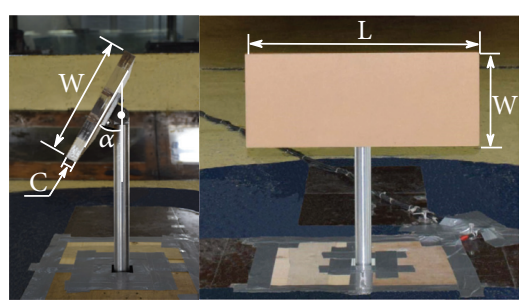

(c)

FIGURE 1: Radar antenna model in wind tunnel test section. (a) Solid plate; (b) porous plate; (c) definition of models.

TABle 1: Test model details.

\begin{tabular}{lcccc}
\hline Antenna type & $L \times W \times C$ & Elevation angle $\left(^{\circ}\right)$ & Porosity rate $(\%)$ & Blocking rate $(\%)$ \\
\hline Solid plate & $25 C \times 10 C \times 1 C$ & 0 & 0 & $<2.5$ \\
Porous plate & $32 C \times 12 C \times 1 C$ & 0 & 38 & $<2.5$ \\
\hline
\end{tabular}

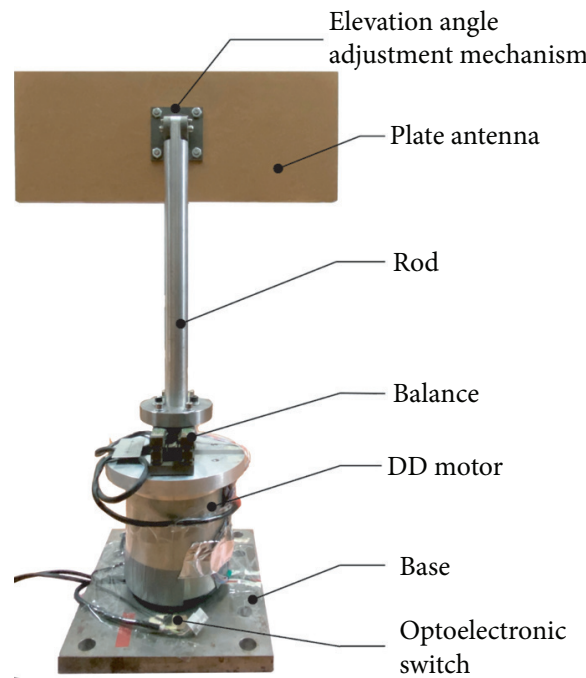

(a)

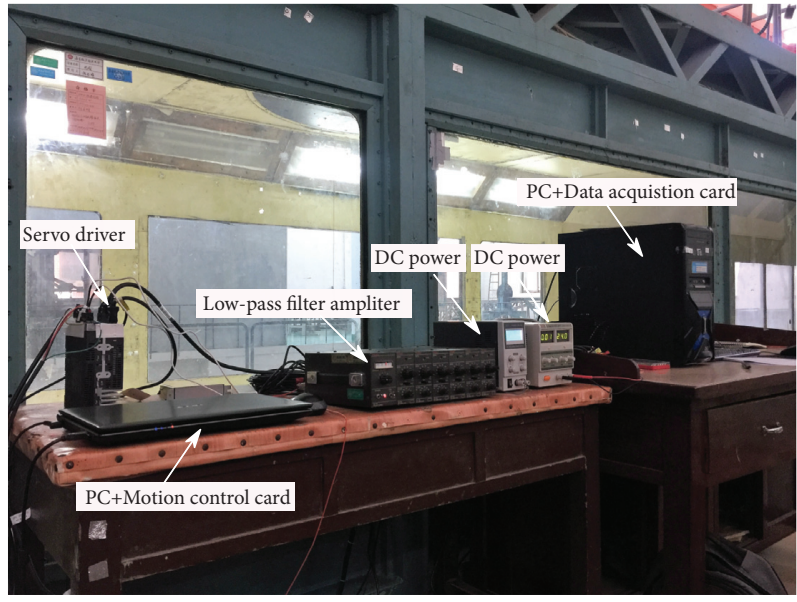

(b)

Figure 2: Wind tunnel dynamic force measurement test platform. (a) Overall driving and model connection method; (b) motion control and data acquisition system.

and $28 \mathrm{~m} / \mathrm{s}$ for the solid plate. The radar antennas are tested at azimuth angles ranging from 0 to $180^{\circ}$ in intervals of $5^{\circ}$.

To study the effects of radar antenna rotation on the aerodynamic characteristics, dynamic test data are also measured. The dynamic wind moment is related to the dimensionless parameter of reduced frequency $K=\mathrm{ND} / \mathrm{V}$, where $\mathrm{N}$ is the rotational angular velocity, $D$ is the antenna rotation diameter, and $\mathrm{V}$ is the flow velocity [10]. The wind tunnel test is carried out in uniform flows of $25 \mathrm{~m} / \mathrm{s}$ for the porous plate and $28 \mathrm{~m} / \mathrm{s}$ for the solid plate, giving values of $K=0.34$ and $K=0.11$, respectively. In this study, the elevation angle remains constant while the radar antenna is rotating. When the model reaches the set speed and rotates stably, an optoelectronic switch installed at an azimuth angle of $0^{\circ}$ sends a signal to trigger the acquisition program to start collecting data at $1 \mathrm{kHz}$ for $6 \mathrm{~s}$. To ensure the accuracy of the wind tunnel test results, monitor the speed during the wind tunnel test, wait for the antenna model to rotate stably at the set rotation speed, and then collect the aerodynamic coefficients for multiple rotation cycles and the aerodynamic coefficient values are displayed over time stable and periodically changing test results.

The output signal of the balance is mixed with vibration noise in the system. The raw data are filtered using a 4 thorder Butterworth low-pass filter, and the cut-off frequency is set to $5 \mathrm{~Hz}$ [21]. The use of a low-pass filter for dynamic data filtering creates a time delay, which will lead to a phase lag in the aerodynamic values. Therefore, zero-phase filtering technology is used to eliminate the phase lag in the system [22]. Figure 3 shows a short segment of the electric signal output by the balance for the raw and filtered test data from the solid plate antenna.

The reference system uses the wind axis system, as shown in Figure 4. The spatial reference system is defined by $X, Y$, 


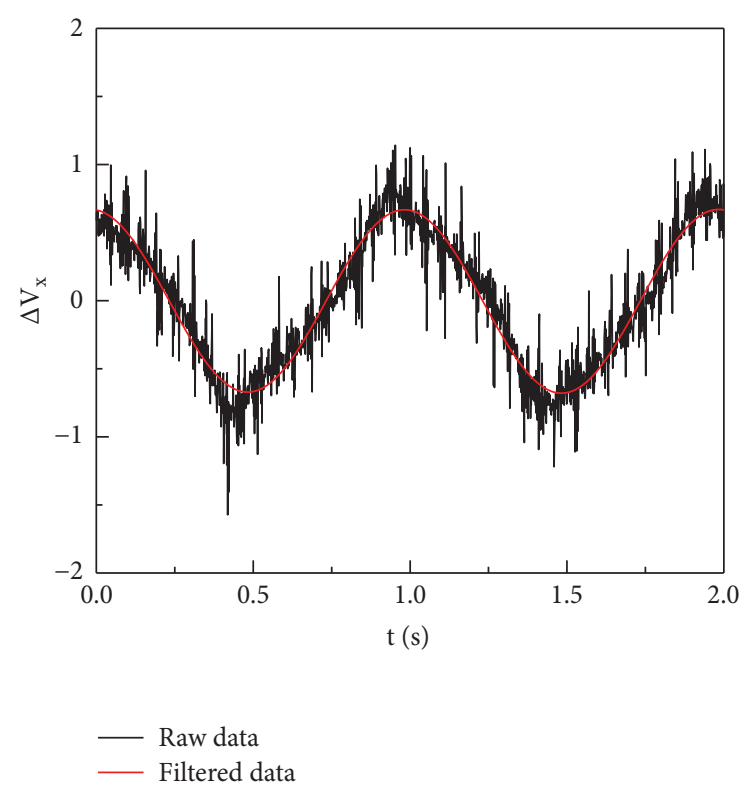

(a)

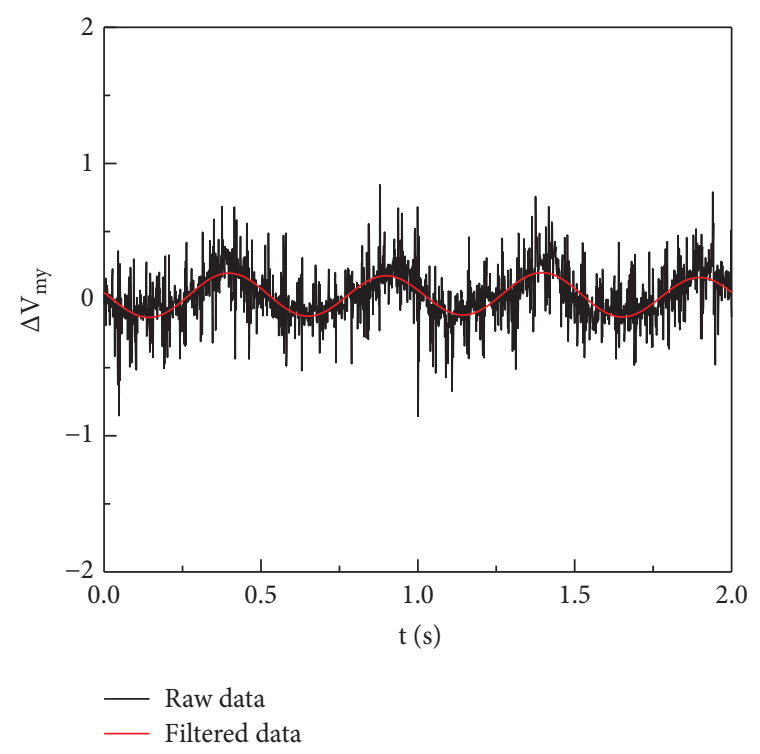

(b)

FiguRE 3: Raw and filtered voltage data of the balance output as a function of time (the zero-point reference set has been deducted). (a) $\Delta V_{x}-t$. (b) $\Delta V_{m y}-t$.

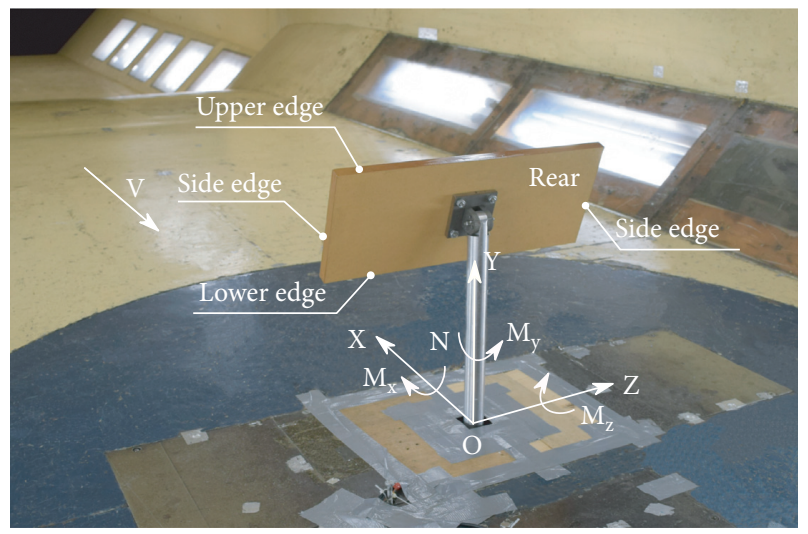

FIGURE 4: Reference system of strain balance (wind axis system).

and $Z$. The $X$ direction is opposite to the wind direction, $Y$ is vertical to the horizontal plane (positive upward), and $Z$ is vertical to the $X O Y$ plane; the reference system follows the right-hand rule, where the drag is opposite to the positive $X$ direction.

In designing a radar antenna servo system that has sufficient structural rigidity, there are five significant aerodynamic forces and moments: drag $\left(F_{x}\right)$, lateral force $\left(F_{z}\right)$, rolling moment $\left(M_{x}\right)$, azimuth moment $\left(M_{y}\right)$, and pitching moment $\left(M_{z}\right)$. The force and moment data of the radar antenna are expressed in dimensionless form using a drag coefficient $C_{x}=F_{x} /\left(0.5 \times \rho \times V^{2} \times S\right)$, lateral force coefficient $C_{z}=F_{z} /\left(0.5 \times \rho \times V^{2} \times S\right)$, rolling moment coefficient $C_{m x}=M_{x} /\left(0.5 \times \rho \times V^{2} \times S \times L\right)$, azimuth moment coefficient $C_{m y}=M_{y} /\left(0.5 \times \rho \times V^{2} \times S \times L\right)$, and pitching moment coefficient $C_{m z}=M_{z} /\left(0.5 \times \rho \times V^{2} \times S \times L\right)$, where $S=L \times W$ is the area of the plate, $L$ and $W$ are the length and width of the plate, $\rho$ is the density of the air, and $V$ is the flow velocity.

\section{Numerical Simulation Approach}

Computational fluid dynamics (CFD) software is used to simulate the antenna flow field distribution. Numerical simulations are carried out for the solid plate antenna and the porous plate antenna.

3.1. Numerical Method. The continuity equation and momentum equations are

$$
\begin{aligned}
\frac{\partial u}{\partial x}+\nabla \cdot(\rho \mu) & =0 \\
\frac{\partial}{\partial x_{j}}\left(\rho u_{i} u_{j}\right) & =-\frac{\partial p}{\partial x_{i}}+\frac{\partial}{\partial x_{j}}\left[\mu \frac{\partial u_{i}}{\partial x_{j}}-\tau_{i j}\right]+S_{i},
\end{aligned}
$$

where $\rho$ is the density; $p$ is the static pressure; $\tau_{i j}$ is the Reynolds stress tensor; $u_{i}$ and $u_{j}$ are the velocity components in the $x_{i}$ and $x_{j}$ directions, respectively; $\mu$ is the dynamic viscosity coefficient; $S_{i}$ contains other related source terms of the model, such as porous medium and user-defined source terms.

The shear-stress transport k- $\omega$ turbulence model is used in numerical simulations [23]. The characteristic of the SST $k-\omega$ model is that the standard $\mathrm{k}-\omega$ model is used in the boundary layer near the wall, $k-\omega$ is used at the edge of the boundary layer and the free shear layer, and a mixed function is used to transition between them. The coefficients are superimposed and written in $k-\omega$ form. In the model 
equation expression, the turbulent kinetic energy equation is as follows:

$$
\frac{\partial}{\partial t}(\rho k)+\frac{\partial}{\partial x_{i}}\left(\rho k u_{i}\right)=\frac{\partial}{\partial x_{j}}\left(\Gamma_{k} \frac{\partial k}{\partial x_{j}}\right)+G_{k}-Y_{k}+S_{k} .
$$

The dissipation rate equation is

$$
\frac{\partial}{\partial t}(\rho \omega)+\frac{\partial}{\partial x_{i}}\left(\rho \omega u_{i}\right)=\frac{\partial}{\partial x_{j}}\left(\Gamma_{\omega} \frac{\partial \omega}{\partial x_{j}}\right)+G_{\omega}-Y_{\omega}+D_{\omega}+S_{\omega}
$$

3.2. Boundary Conditions. The computational domain and antenna model are established on a $1: 1$ scale with respect to the wind tunnel test section and the antenna test model size. The antenna model is positioned in the middle of the computational domain, $150^{\circ} \mathrm{C}$ from both the entrance and exit, and the blockage rate of the model is less than $2 \%$. Simulations are performed with a uniform inflow $(28 \mathrm{~m} / \mathrm{s}$ for solid plate antenna; $25 \mathrm{~m} / \mathrm{s}$ for porous plate antenna) corresponding to the flow velocity in the wind tunnel tests. As shown in Figure 5(a), the computational domain is divided into a stationary domain and a rotation domain. A refinement domain is added to the stationary domain around the antenna. The rotation domain is used to realize the azimuthal rotation of the antenna. Data transmission between different computing domains is realized by a sliding mesh dynamic-static interface. The computational domain is divided into unstructured meshes, and the cells near the antenna model are refined. The maximum mesh size of the stationary domain, refinement, and rotating domain is $80 \mathrm{~mm}, 20 \mathrm{~mm}$, and $10 \mathrm{~mm}$; the minimum mesh size of the stationary domain, refinement, and rotating domain is default, $2 \mathrm{~mm}$, and $1 \mathrm{~mm}$. Through a mesh-independence verification, the total number of cells is determined on the premise of ensuring the accuracy of the numerical simulations and the integrity of the flow field characteristics. As an example to verify the mesh independence, the results for the solid plate antenna are presented in Table 2. Based on these methods, the number of grids of solid and porous plate antennas is about 8 million and 10 million, respectively. In addition, the inlet boundary adopts the velocity inlet condition; the outlet boundary adopts the pressure outlet condition with a relative pressure of $0 \mathrm{~Pa}$; the nonslip condition is applied at the other boundaries, as shown in Figure 5(b). The SIMPLE algorithm is adopted for the pressure-velocity coupling. The first-order implicit scheme for the unsteady time derivative term is applied. The standard wall function is used in numerical simulation.

The numerical simulations are divided into two parts: steady numerical simulations and unsteady numerical simulations. In the steady numerical simulations, the antenna azimuth angle $\beta=0-180^{\circ}$ and $\Delta=10^{\circ}$ (total 19 positions), and the iteration number in each simulation is 3000 . In the unsteady numerical simulations, when the reduced frequency $K=0.11$, the calculation time step is $0.002778 \mathrm{~s}$; when the reduced frequency $K=0.34$, the calculation time step is $0.001389 \mathrm{~s}$; the corresponding azimuth angle is $1^{\circ}$. To ensure the accuracy of the CFD results, during the numerical simulation, monitor the residual value and the aerodynamic coefficient value. For steady simulations, observe whether the velocity residual value is below $10^{-4}$ and the aerodynamic coefficient remains stable; for unsteady simulations, observe the change of the aerodynamic coefficient value with the calculation step. When it shows a stable periodic change, continue to calculate multiple rotation cycles to obtain the CFD result with a stable periodic change of the aerodynamic coefficient with respect to time. The aerodynamic coefficient components decomposed by the model in the wind axis system, that is, $C_{x}, C_{z}, C_{m x}, C_{m y}$, and $C_{m z}$, are monitored and recorded. As the antenna model is symmetric about the XOY plane, the mean, maximum, and root mean square (RMS) values of the steady aerodynamic coefficients of the antenna are the same in the ranges $\beta=0-180^{\circ}$ and $\beta=180-360^{\circ}$ without considering the direction of the force and moment.

\section{Results and Discussion}

\subsection{Effects of Rotation Motion on Aerodynamic Characteristics of Solid Plate Antenna}

4.1.1. Aerodynamic Coefficient of Solid Plate Antenna at Rest. Figure 6 shows the wind tunnel test results and numerical simulation results of aerodynamic coefficient with respect to the azimuth angle when the antenna is at rest. It can be seen that the numerical simulation results are in relatively good agreement with the wind tunnel test results. Without considering the aerodynamic force and moment direction, the simulation results of the aerodynamic coefficients for $\beta=0-90^{\circ}$ and $\beta=90-180^{\circ}$ are symmetric about the position $\beta=90^{\circ}$. The test results show that the positive peaks of $C_{x}$ and $C_{m z}$ appear near $\beta=0^{\circ}$ and $180^{\circ}$ and the negative peaks appear at $\beta=90^{\circ}$. The simulation results have positive peaks of $C_{x}$ and $C_{m z}$ near $\beta=40^{\circ}$ and $135^{\circ}$, which indicates that the numerical simulation method underestimates the size of $C_{x}$ and $C_{m z}$ when the antenna is located at the maximum windward position $\left(\beta=0^{\circ}\right.$ and $\left.180^{\circ}\right)$.

\subsubsection{Aerodynamic Coefficient of Solid Plate Antenna during} Azimuthal Rotation. Under the effect of wind loading, accurate acquisition of the maximum and RMS value of the antenna aerodynamic coefficient is particularly important for antenna servo system design and to ensure structural stiffness and strength. Larger peak values of the aerodynamic coefficients will place higher requirements on the ability of the servo system to resist variable loads, and the volume and weight of the servo motor will increase accordingly. These parameters ultimately determine the reliability and energy consumption of the equipment [12].

Figure 7 shows the wind tunnel test results and numerical simulation results of aerodynamic coefficient with respect to the azimuth angle when the antenna rotates in azimuth. As shown in the figure, the test results with respect to the azimuth angle are in good agreement with the simulation results. $C_{x}$ and $C_{m y}$ do not attain the maximum values at the maximum windward region of the antenna, and $C_{z}, C_{m x}$, and $C_{m y}$ are not equal to zero. Note that, except for 


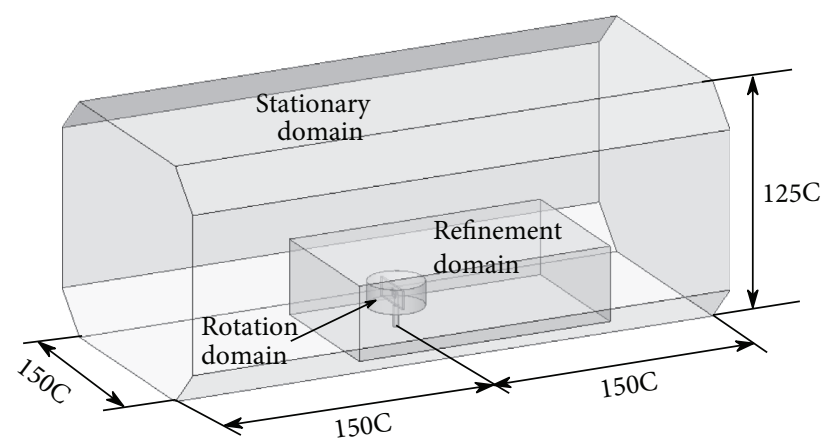

(a)

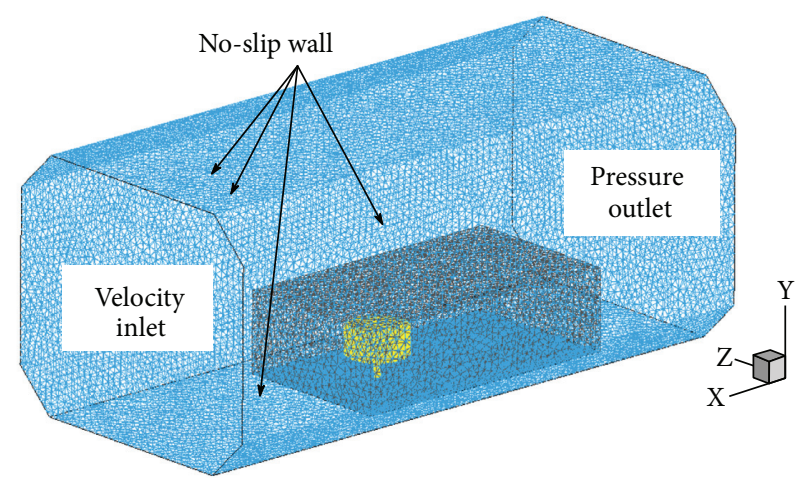

(b)

Figure 5: Computational domain and mesh division. (a) Computational domain; (b) mesh division around the model.

TABLE 2: CFD mesh-independence verification for $V=28 \mathrm{~m} / \mathrm{s}$ with $K=0.11$. Data in parentheses are the percentage differences in aerodynamic coefficient values between the current number and the finest mesh number (solid plate antenna).

\begin{tabular}{lccc}
\hline Mesh number & 16 million & 8 million & 4 million \\
\hline$C_{x}$ & 2.33 & $2.34(0.4 \%)$ & $2.33(0 \%)$ \\
$C_{z}$ & -1.24 & $-1.23(0.8 \%)$ & $-1.25(-0.8 \%)$ \\
$C_{m x}$ & -0.87 & $-0.87(0 \%)$ & $-0.87(0 \%)$ \\
$C_{m y}$ & 0.11 & $0.11(0 \%)$ & $0.10(-9 \%)$ \\
$C_{m z}$ & 1.53 & $1.54(0.7 \%)$ & $1.54(0.7 \%)$ \\
\hline
\end{tabular}

$C_{m y}$, the peak values of the aerodynamic coefficients are overestimated by the numerical method, whereas the RMS values of the aerodynamic coefficients are underestimated. With the exception of the RMS of $C_{x}$, the simulation results for the aerodynamic coefficient RMS values are slightly smaller than the test results.

There are some differences in the peak values given by the numerical simulations and the wind tunnel tests. This is because the turbulence model cannot accurately express the pressure of vortices and only provides the statistical characteristics of this quantity [18].

4.1.3. Comparison of Aerodynamic Coefficients between Stationary and Azimuthal Rotation of Solid Plate Antenna. Figure 8 shows the wind tunnel test results of the aerodynamic coefficients when the antenna is stationary and the azimuthal rotation in the range of $\beta=0-360^{\circ}$. It can be noted from this figure that the aerodynamic coefficients of the antenna in azimuthal rotation are continuous and smooth with respect to the azimuth angle; similar phenomena were reported by Lombardi [3].

Table 3 gives the wind tunnel test results for the mean, maximum, and RMS values of the aerodynamic coefficients of solid plate antennas at rest and when rotating. Quantitative analysis indicates that the mean, maximum, and RMS values increase when rotating compared with when the antenna is at rest. $\mid C_{x \_}$mean|, $\mid C_{m y \_}$mean $\mid$, and $\mid C_{m z}$ mean $\mid$ increase by $12.4 \%, 500 \%$, and $13.3 \%$, respectively; $\left|C_{x}\right| \_\max$, $\left|C_{m y}\right| \_$max, and $\left|C_{m z}\right| \_$max increase by $41.6 \%, 15.0 \%$, and
$47.3 \%$, respectively; $C_{x_{-}} \mathrm{rms}, C_{m y \_} \mathrm{rms}$, and $C_{m z} \mathrm{rms}$ increase by $19.0 \%, 20.0 \%$, and $19.1 \%$, respectively. Therefore, changing from a static antenna to azimuthal rotation places a greater wind load on the whole structure. Moreover, the radar antenna is subject to a periodic alternating load for a long time, which will reduce the service life of the servo motor and increase the shutdown rate of the radar antenna.

When the antenna is stationary, the aerodynamic coefficients $C_{x}$ and $C_{m z}$ have positive peaks at $\beta=180^{\circ}$ and negative peaks at $\beta=90^{\circ} ; C_{z}$ and $C_{m x}$ have positive peaks at $\beta=45^{\circ}$ and negative peaks at $\beta=135^{\circ}$, and the opposite is true for $C_{m y}$. However, the rotation motion will lead to some advance or lag in the azimuthal position corresponding to the peak aerodynamic coefficient of the solid plate antenna. Therefore, the effect of the aerodynamic load on the aerodynamic characteristics of radar antennas with azimuthal rotation cannot be ignored. Otherwise, it would be very difficult to design a lightweight antenna servo system and transmission mechanism under the premise of ensuring stability, flexibility, safety, reliability, and a long service life [24].

4.1.4. Extraction of Surface Pressure Coefficient of Solid Plate Antenna. As the size and range of application of radar antennas increase, the vibrations caused by wind loading as the antenna rotates have an increasingly prominent effect on their stability and electrical performance. For a rotating radar antenna, the wind load changes periodically, which will lead to periodic vibrations of the antenna and periodic changes in the aerodynamic coefficients. Jin and $\mathrm{Xu}$ [25] reported that, under wind loading, the antenna moment mainly comes from the pressure rather than the shear stress and is caused by the negative pressure region formed by the shedding vortex on the leeward side, which results in uneven stress on the antenna. Therefore, it is necessary to predict and analyze the wind pressure distribution on the antenna surface.

In Figure 9, A and B represent the front and back of the solid plate antenna, respectively. Six monitoring points are arranged near the edges of both sides of each surface, giving a total of 12 monitoring points. These can be used to measure the changes in the antenna surface pressure coefficient with 

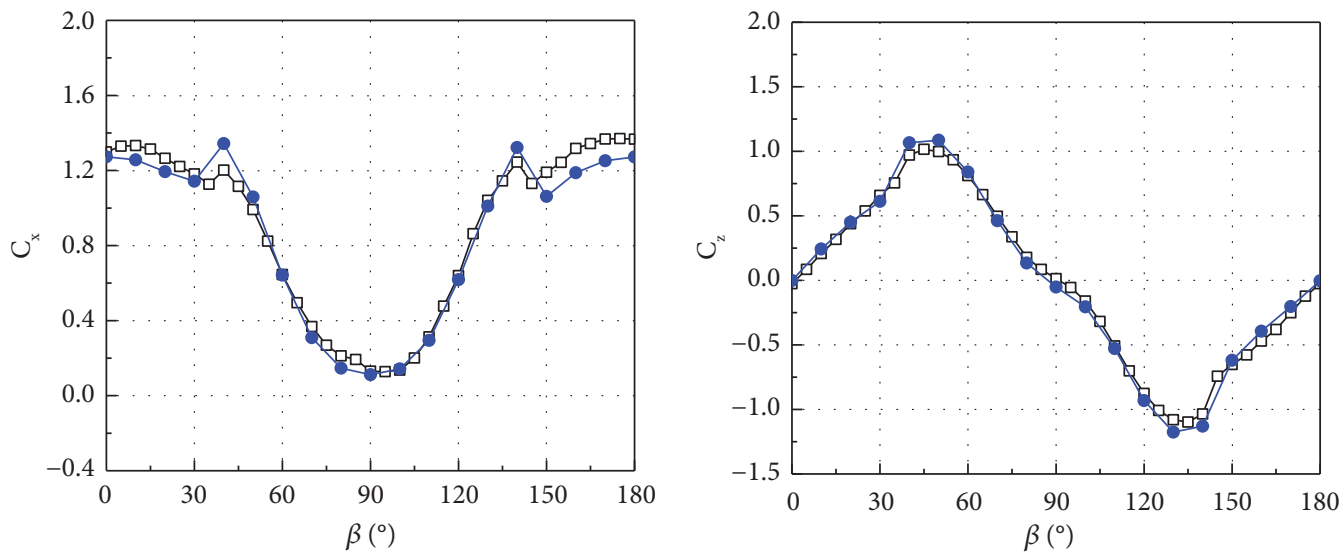

$\rightarrow-$ Exp._stationary

- CFD_stationary

$\rightarrow$ Exp._stationary

$\rightarrow$ CFD_stationary

(a)

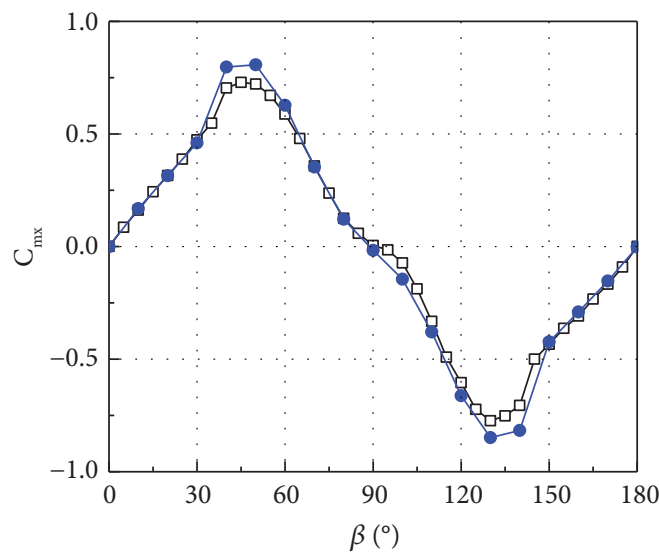

(b)

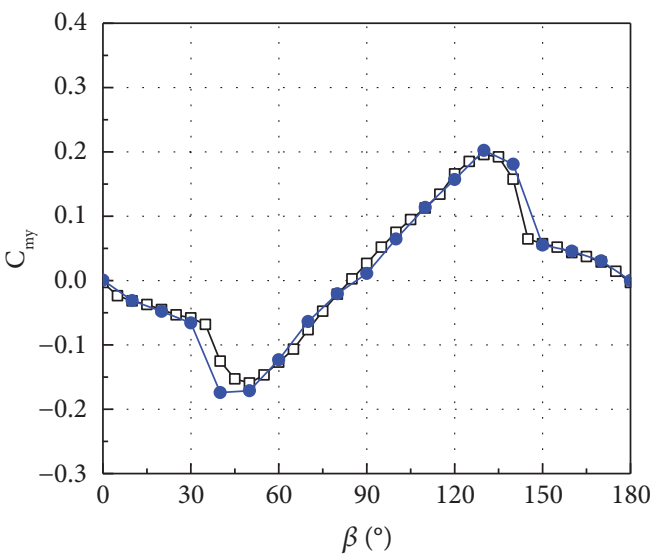

$\rightarrow-$ Exp._stationary $\quad \rightarrow-$ Exp._stationary

$\bullet$ CFD_stationary

$\rightarrow$ CFD_stationary

(c)

(d)

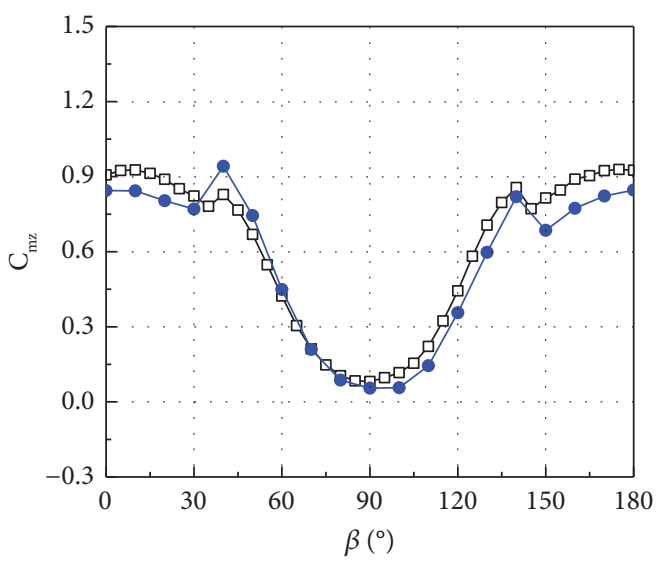

$\rightarrow \square$ Exp._stationary

$\rightarrow$ CFD_stationary

(e)

FIGURE 6: Wind tunnel test results and numerical simulation results of aerodynamic coefficient with respect to the azimuth angle when the antenna is at rest (solid plate, $\beta=0-180^{\circ}$ ). (a) $C_{x}-\beta$, (b) $C_{z}-\beta$, (c) $C_{m x}-\beta$, (d) $C_{m y}-\beta$, and (e) $C_{m z}-\beta$. 


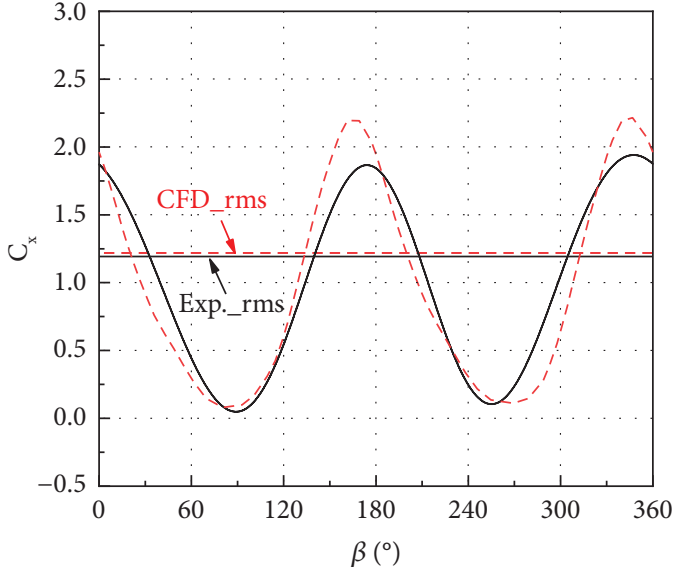

_ Exp._rotation

- - CFD_rotation

(a)

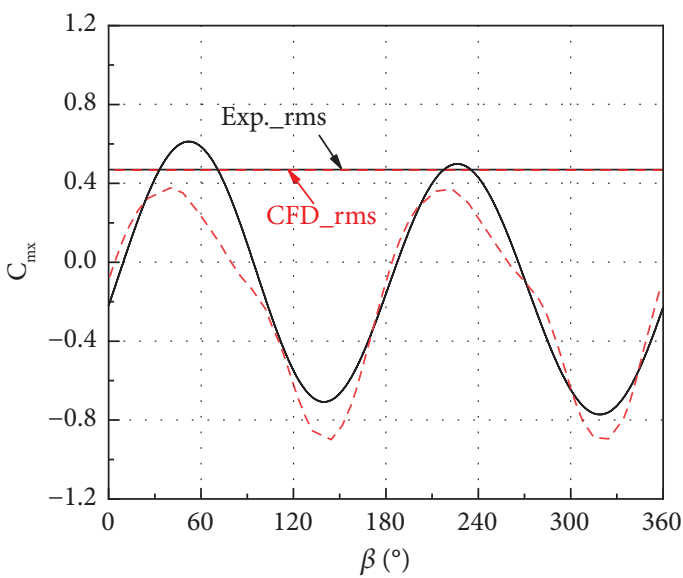

- Exp._rotation - - - CFD_rotation

(c)

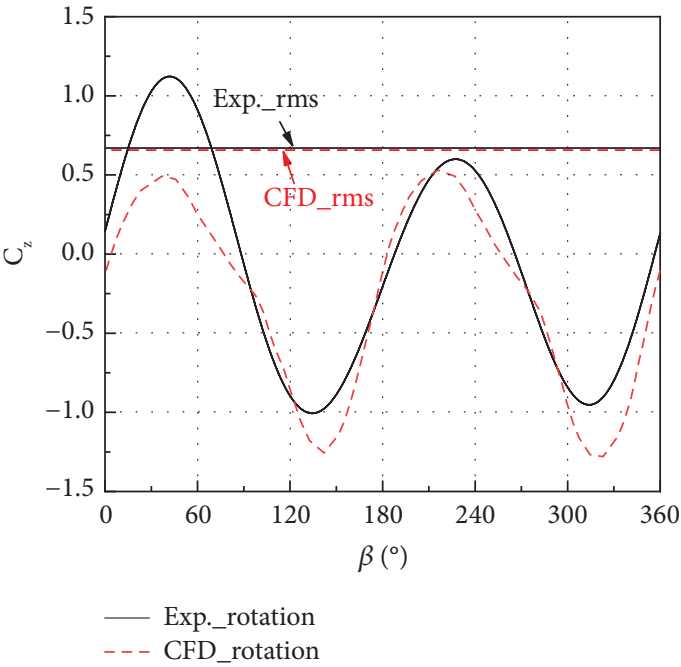

(b)

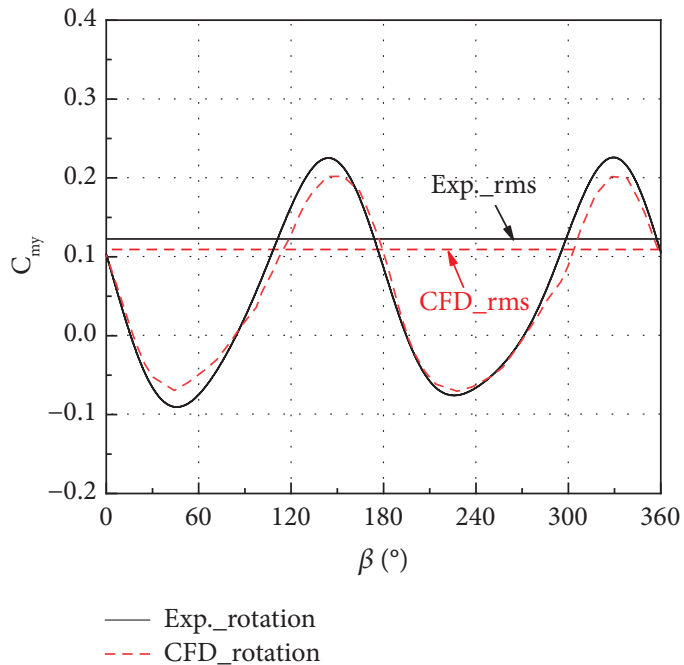

(d)

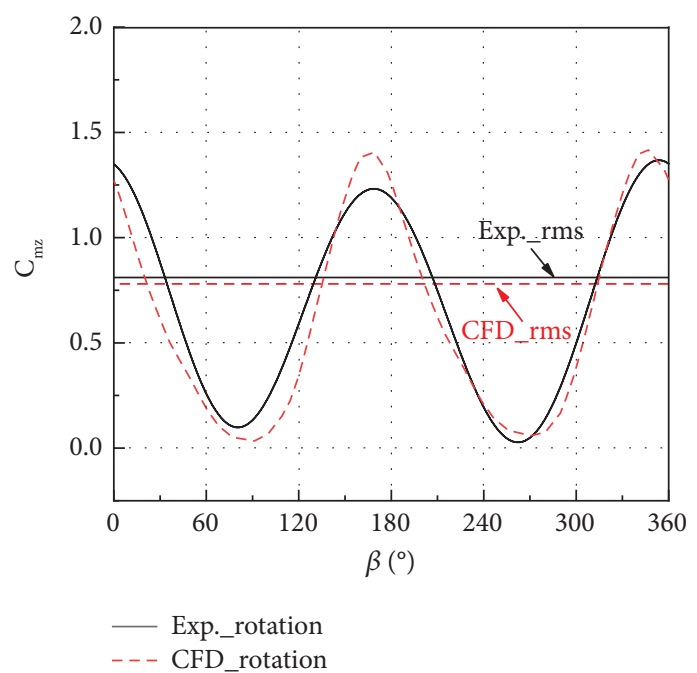

(e)

Figure 7: Wind tunnel test results and numerical simulation results of aerodynamic coefficient with respect to azimuth angle when the antenna rotates in azimuth (solid plate, $K=0.11$ ). (a) $C_{x}-\beta$, (b) $C_{z}-\beta$, (c) $C_{m x}-\beta$, (d) $C_{m y}-\beta$, and (e) $C_{m z}-\beta$. 


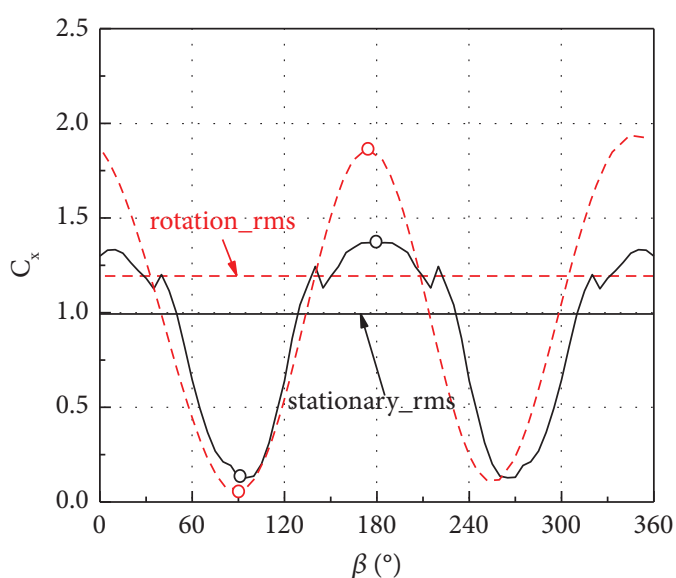

— Exp._stationary

- - Exp._rotation

(a)

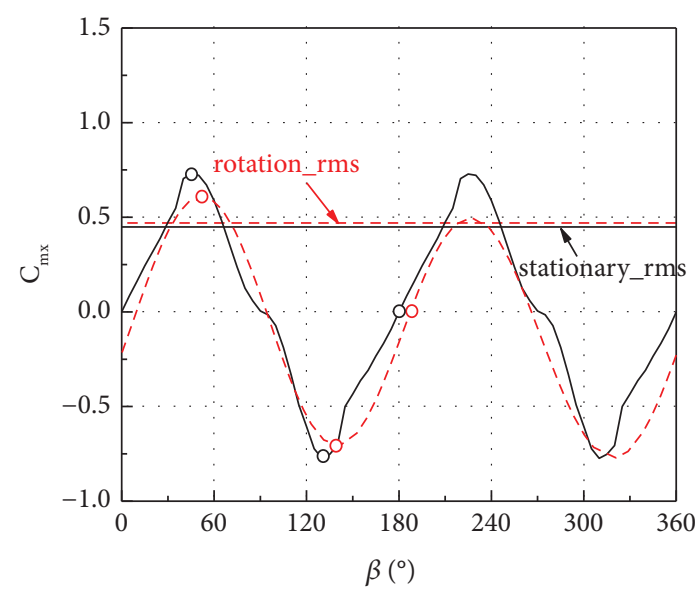

- Exp._stationary

- - - Exp._rotation

(c)

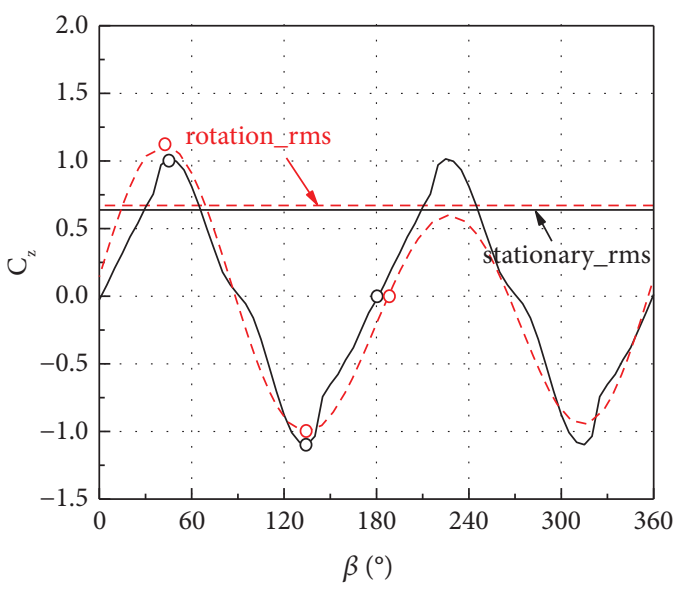

_ Exp._stationary

- - - Exp._rotation

(b)

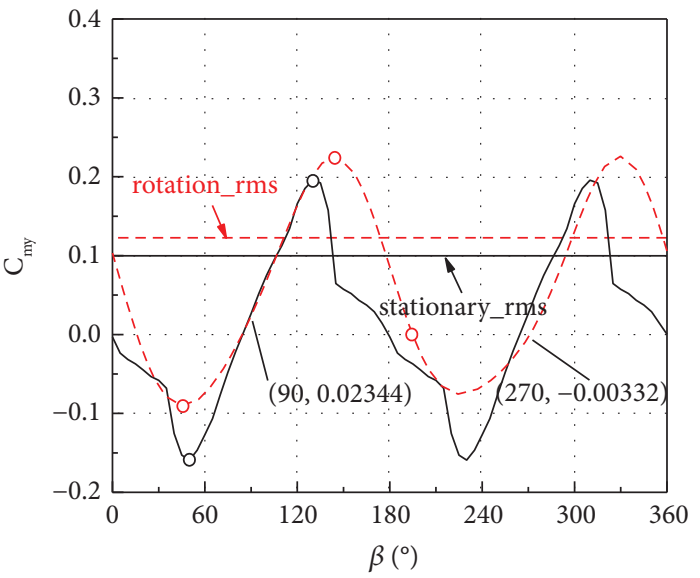

_ Exp._stationary

- - - Exp._rotation

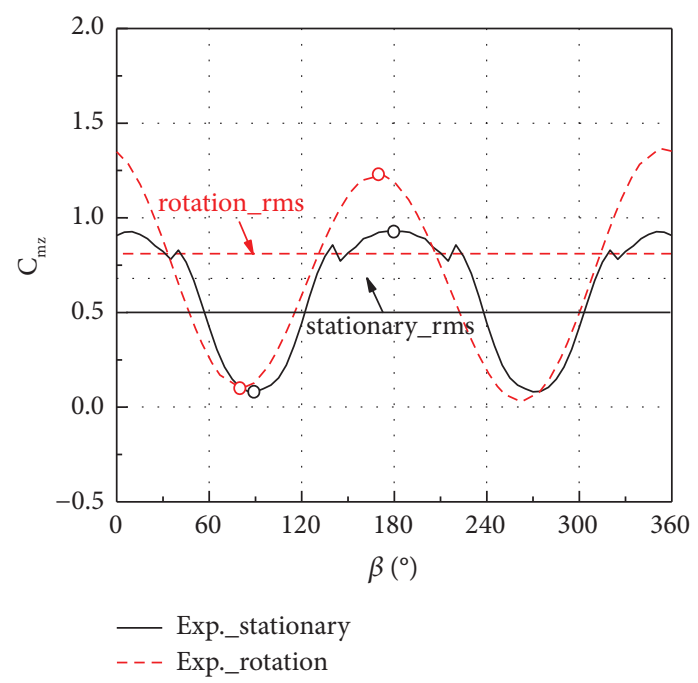

(e)

Figure 8: Wind tunnel test results and numerical simulation results of aerodynamic coefficients with respect to the azimuth angle when the antenna is stationary and azimuthal rotation (solid plate), (a) $C_{x}-\beta$, (b) $C_{z}-\beta$, (c) $C_{m x}-\beta$, (d) $C_{m y}-\beta$, and (e) $C_{m z}-\beta$. 
TABLE 3: The mean, maximum, and RMS values of aerodynamic coefficients for the solid plate antenna when the antenna is stationary and azimuthal rotation (the direction is not included in these results).

\begin{tabular}{|c|c|c|c|c|}
\hline \multicolumn{2}{|c|}{ Solid plate } & \multicolumn{3}{|c|}{ Exp. } \\
\hline & & Mean & Maximum & RMS \\
\hline \multirow{5}{*}{ Stationary } & $C_{x}$ & 0.89 & 1.37 & 1.00 \\
\hline & $C_{z}$ & 0.02 & 1.01 & 0.64 \\
\hline & $C_{m x}$ & 0.00 & 0.73 & 0.45 \\
\hline & $C_{m y}$ & 0.01 & 0.20 & 0.10 \\
\hline & $C_{m z}$ & 0.60 & 0.93 & 0.68 \\
\hline \multirow{5}{*}{ Azimuthal rotation } & $C_{x}$ & 1.00 & 1.94 & 1.19 \\
\hline & $C_{z}$ & 0.06 & 1.12 & 0.67 \\
\hline & $C_{m x}$ & 0.09 & 0.77 & 0.47 \\
\hline & $C_{m y}$ & 0.06 & 0.23 & 0.12 \\
\hline & $C_{m z}$ & 0.68 & 1.37 & 0.81 \\
\hline
\end{tabular}

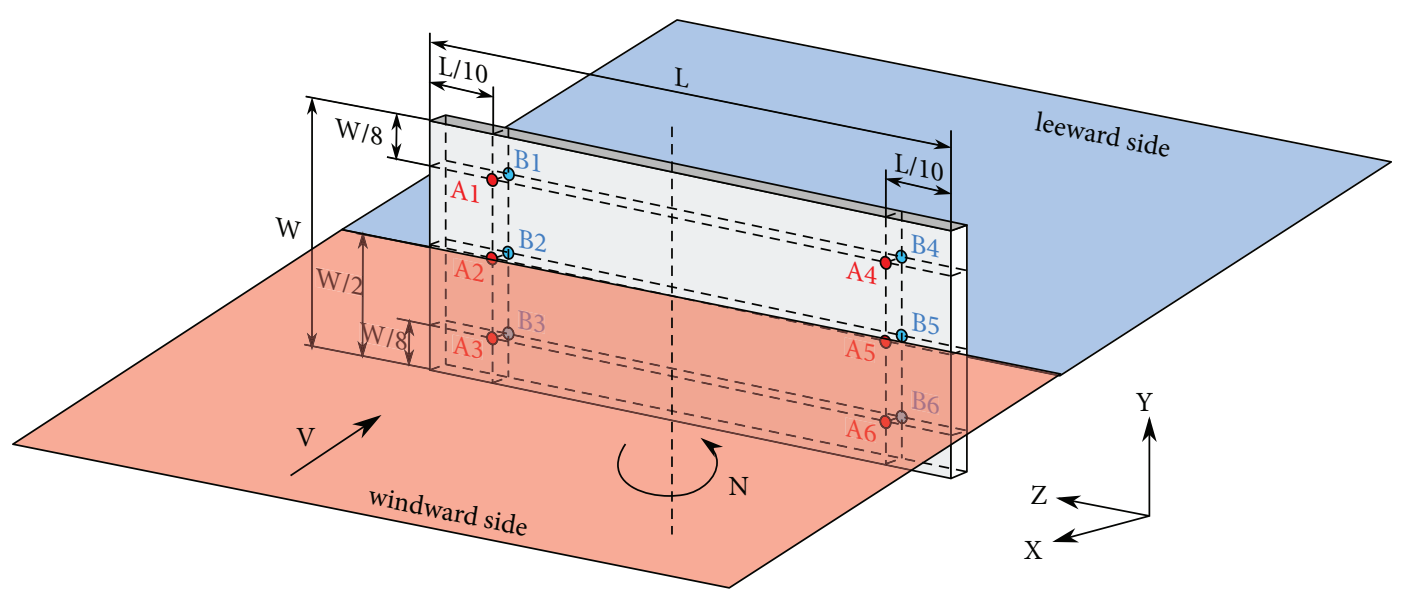

FIGURE 9: Schematic diagram of pressure monitoring points on the surface of a solid plate antenna (red region represents the windward side of the antenna, blue region represents the leeward side of the antenna, and rotation is counterclockwise).

azimuth angle. The pressure results at each monitoring point, as obtained from numerical simulations, are given in the dimensionless form, and the pressure coefficient $\left(C_{p}\right)$ of each monitoring point is calculated by $C_{p}=\left(p_{i}-p_{r}\right) / 0.5 \rho V^{2}$, where $p_{i}$ is the pressure measured at monitoring point $i, p_{r}$ is the pressure of the free flow, $\rho$ is the air density, and $V$ is the flow velocity.

Figure 10 shows the variation of the pressure coefficients with azimuth angle at each monitoring point when the solid plate antenna is stationary. It can be seen that the pressure coefficient at each monitoring point changes irregularly with the azimuth. The degree of fluctuation in the pressure coefficient at each monitoring point on the antenna surface is almost the same. Besides, the existence of the antenna rod leads to the pressure distribution at the monitoring points on both sides of the front of the antenna, which is more uniform than that on the back of the antenna, as shown in Figure 11.

Figure 12 shows the variation of the pressure coefficient at each monitoring point on the antenna surface with respect to the azimuth angle. When the monitoring point is located on the windward side, the pressure coefficient is positive, and the positive pressure value at monitoring point 2 is higher than those at monitoring points 1 and 3 near the upper and lower edges. When the monitoring point is located on the leeward side, the pressure coefficient is negative, and the negative pressure value at monitoring point 2 is lower than those of monitoring points 1 and 3 near the upper and lower edges. For $\beta=83-265^{\circ}$, the pressure coefficients at monitoring points $\mathrm{B} 1, \mathrm{~B} 2$, and $\mathrm{B} 3$ are greater than those at monitoring points A1, A2, and A3. For $\beta=90-270^{\circ}$, the pressure coefficients at monitoring points $\mathrm{B} 4, \mathrm{~B} 5$, and $\mathrm{B} 6$ are greater than those at monitoring points A4, A5, and A6. Different from the variation in the aerodynamic coefficient with azimuth angle, the variation in the pressure coefficients does not exhibit symmetry over one rotation period, which explains why the two positive peak values (negative peak values) are different in the variation of the moment coefficient with azimuth angle during antenna rotation. The pressure coefficients at the front and back monitoring points have obvious differences depending on the azimuth angle.

Figure 13 shows the vorticity nephogram and pressure isosurface nephogram, the black solid line indicates that the pressure value is positive, the black dotted line indicates that the pressure value is negative, and color cloud images represent vorticity. It can be seen from the figure that the pressure values in the vorticity intensive area are negative, and the location of the negative pressure area is greatly 


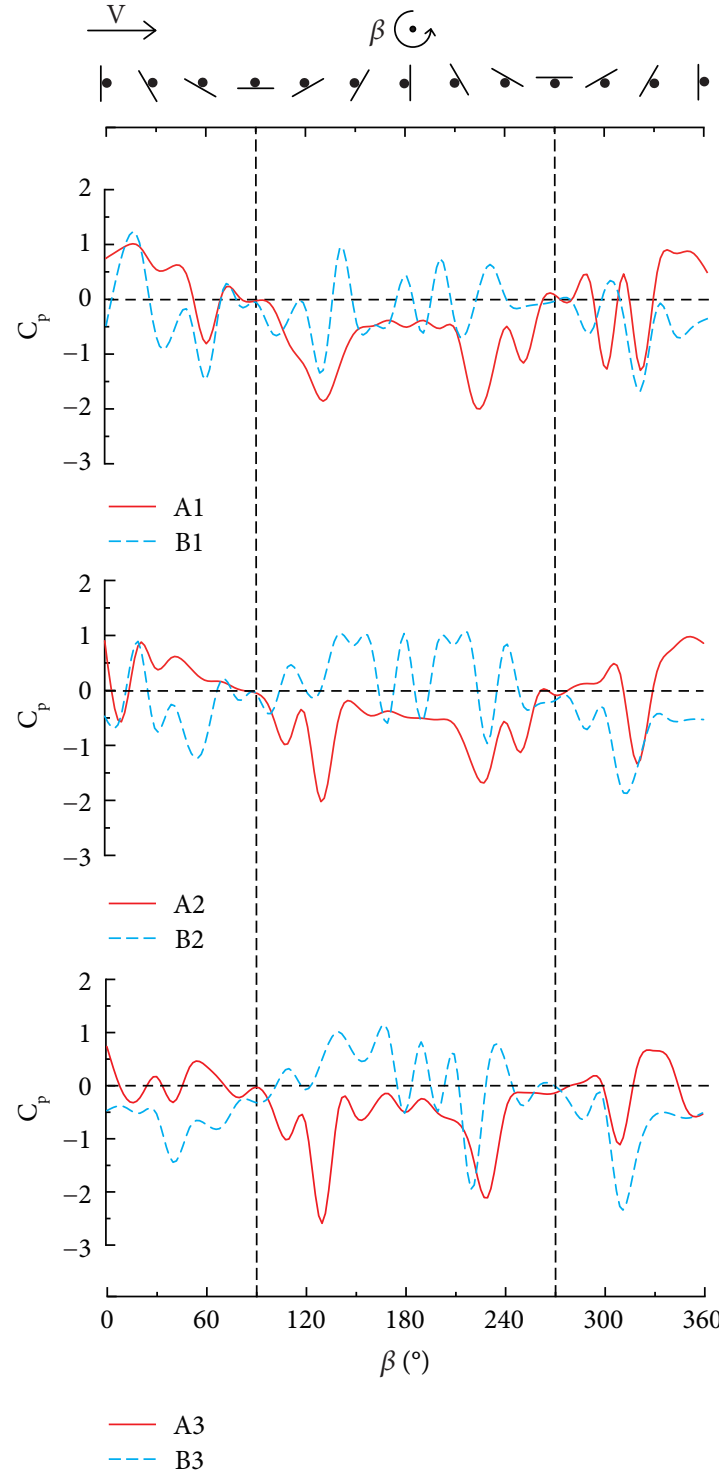

(a)

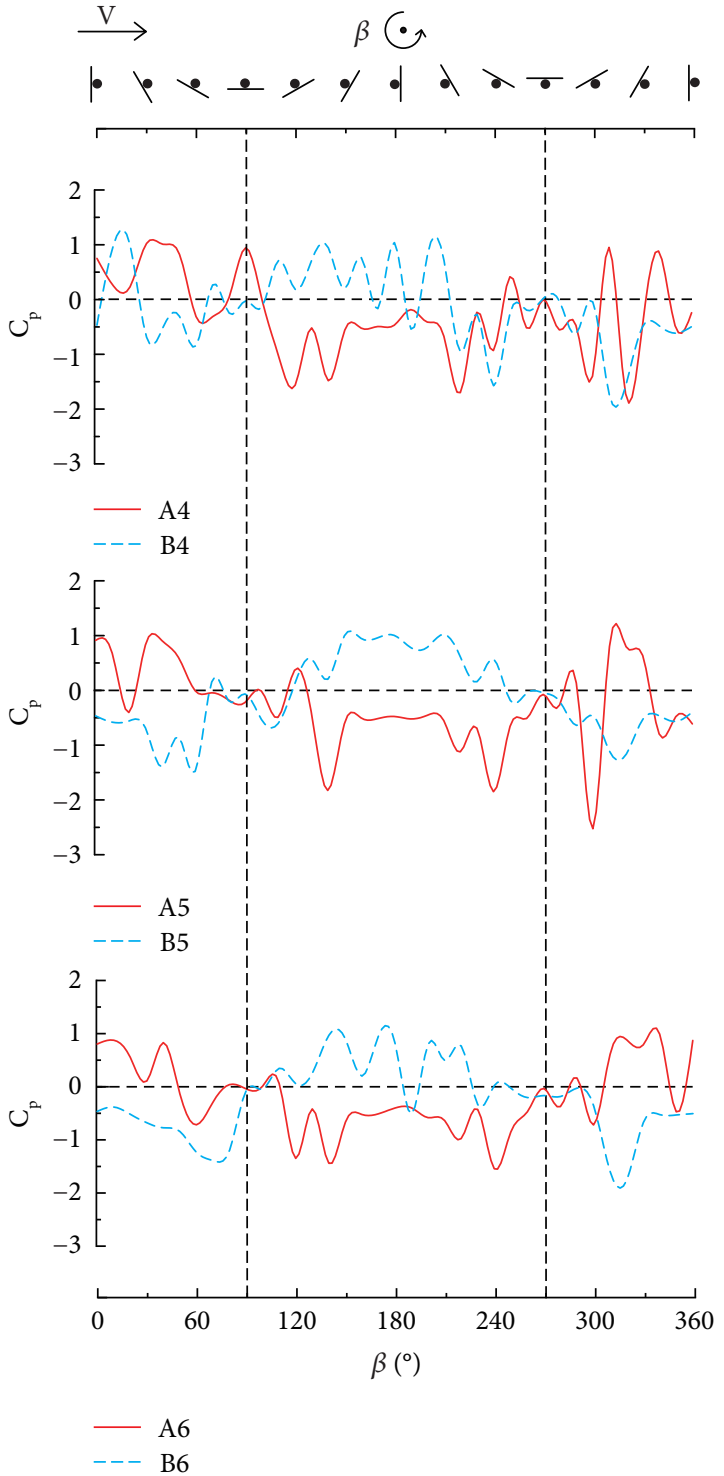

(b)

Figure 10: Pressure coefficients of the surface monitoring points with respect to azimuth angle when the solid plate antenna is stationary, $\beta=0-360^{\circ}$, and $\Delta=10^{\circ}$. (a) Monitoring points 1,2 , and 3 ; (b) monitoring points 4,5 , and 6 (A represents the front of the antenna and $\mathrm{B}$ represents the back of the antenna).

affected by the location of the vorticity intensive area. In addition, shedding vortices are formed on both sides of the antenna and propagate downstream. The area where the shedding vortices are generated is dark red. The results show that the vortices in the flow field around the antenna do not become completely mixed as the antenna rotates, which results in an uneven pressure distribution on the front and back of the antenna. Over one rotation period, the positive peak pressure coefficients of each monitoring point on the windward side are almost the same, whereas the negative peak pressure coefficients of each monitoring point on the leeward side are slightly different. This indicates that the shedding vortex generated by the antenna edge has little influence on the pressure distribution of the windward side but has a greater impact on the leeward side pressure distribution.
It can be seen from Figure 8(d) that when the antenna is stationary at $\beta=90^{\circ}$ and $270^{\circ}$, its azimuth moment coefficient is zero; when the antenna rotates, the aerodynamic coefficient is positive at $\beta=90^{\circ}$ and negative at $\beta=270^{\circ}$. Figure 10 shows that when $\beta=90^{\circ}$, the pressure coefficients of points $\mathrm{A} 1$ and $\mathrm{B} 1$, points $\mathrm{A} 2$ and $\mathrm{B} 2$, and points $\mathrm{A} 3$ and $\mathrm{B} 3$ are negative, and the pressure differences at these positions are less than zero. As can be seen from Figure 10, when $\beta=90^{\circ}$, the pressure coefficients of points $\mathrm{A} 1$ and $\mathrm{B} 1, \mathrm{~A} 2$ and $\mathrm{B} 2$, and $\mathrm{A} 3$ and $\mathrm{B} 3$ are close to zero. The pressure difference between the front and back surfaces is about zero. Although the pressure coefficients of point $\mathrm{B} 4$, points $\mathrm{A} 5$ and $\mathrm{B} 5$, and points A6 and B6 are also less than zero and the pressure difference between the front and back surfaces is almost equal to zero, the positive pressure value at point $\mathrm{A} 4$ is 


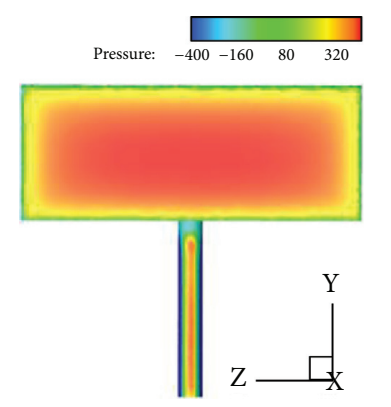

(a)

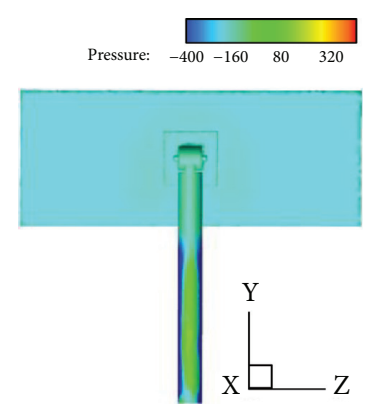

(e)

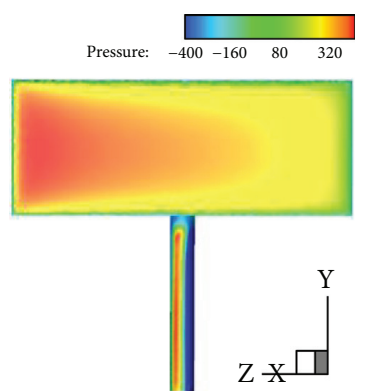

(b)

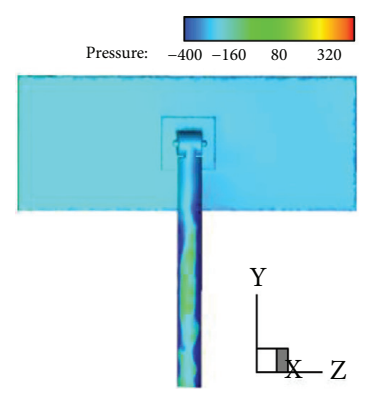

(f)

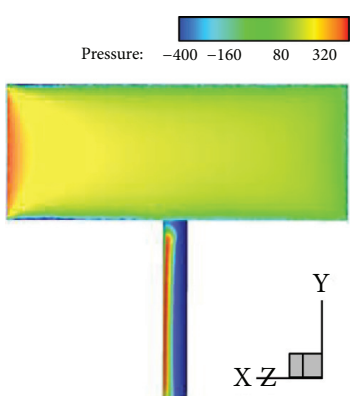

(c)

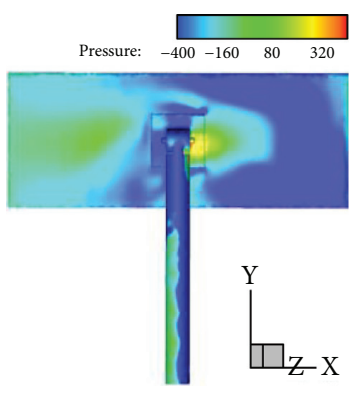

(g)

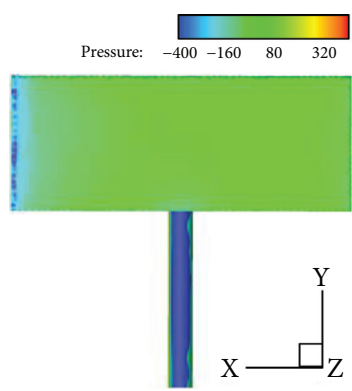

(d)

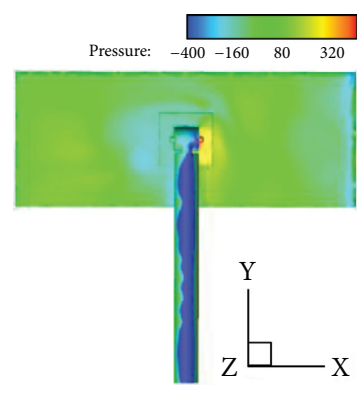

(h)

Figure 11: Nephogram diagram of pressure distribution on the front and back of the antenna. (a)-(d) The front of the antenna; (e)-(h) the back of the antenna. (a) $\beta=0^{\circ}$, (b) $\beta=30^{\circ}$, (c) $\beta=60^{\circ}$, (d) $\beta=90^{\circ}$, (e) $\beta=0^{\circ}$, (f) $\beta=30^{\circ}$, (g) $\beta=60^{\circ}$, and (h) $\beta=90^{\circ}$.

obviously large, resulting in a positive azimuth moment of the antenna, so $C_{m y}$ is great than zero. By $\beta=270^{\circ}$, the differential pressure coefficients of points $A 1$ and $B 1$, points $\mathrm{A} 2$ and $\mathrm{B} 2$, and points $\mathrm{A} 3$ and $\mathrm{B} 3$ are less than zero, and the antenna produces a reverse azimuth moment, so the azimuth moment coefficient is negative. When the antenna is near $\beta=145^{\circ}$, the surface pressure coefficients at the front monitoring points (A1, A2, and A3) reach the minimum values, and the pressure differences at monitoring points $\mathrm{B} 1$, $\mathrm{B} 2$, and $\mathrm{B} 3$, corresponding to the back, reach the maximum values; the azimuth moment coefficient also attains the maximum.

From the perspective of quantitative analysis, the surface pressure coefficients of monitoring points $\mathrm{A} 1-\mathrm{A} 3$ and $\mathrm{B} 1-\mathrm{B} 3$ change greatly with the azimuth angle, with $-3<C_{p}<2$; in contrast, the surface pressure coefficients of monitoring points $\mathrm{A} 4-\mathrm{A} 6$ and $\mathrm{B} 4-\mathrm{B} 6$ change little with azimuth, with $-1<C_{p}<1$. As the antenna rotates, both the front and back of the antenna are on the windward side for some time. However, because there is an elevation angle adjustment mechanism and a rod on the back of the antenna, the variations in the surface pressure coefficients of the monitoring points near the two side edges of the antenna are not the same. This can also be seen from Figure 11.

4.1.5. Extraction of Vortex Structures of Solid Plate Antenna. As the pressure in a vortex is lowest at its core and increases with distance from the core, the position distribution of the vortices will affect the aerodynamic coefficient of the antenna by affecting the pressure distribution around and on its surface. Therefore, analysis of the vortex core positions in the flow field can reveal the formation mechanism of different pressure distributions and reveal the differences in aerodynamic characteristics between static and rotating antennas [26].

Figure 14 shows the distribution of the spatial vortex core and the multisection pressure nephogram diagram of the $Y O Z$ pressure field. It can be seen from the figure that the pressure value at the location of the vortex core is low. To further explore the reasons for the discrepancies in the antenna surface pressure distribution, Figure 15 shows the distribution of vortex core positions in the flow field of a solid plate antenna at $\beta=0^{\circ}, 30^{\circ}, 60^{\circ}$, and $90^{\circ}$. From Figures $15(\mathrm{e})$ and $15(\mathrm{~g})$, combined with Figure 12, when a continuous vortex core track appears near the antenna, the surface pressure distribution is greatly affected. It can be seen from Figures 15(b) and 15(f) that although the vortex core is close to the antenna, the continuous vortex core track has a greater impact on the antenna surface pressure distribution. The effects of the vortex core on the antenna surface pressure distribution are not obvious because the vortex core track is scattered.

As the pressure is proportional to the distance from the vortex core, the distribution of vortex cores around the antenna has a large effect on the surface pressure. In Figure 15, the region with a high density of vortex cores around the antenna is roughly illustrated by the blue square. Except in Figure 15(a), the vortex cores near the antenna are mainly distributed in one or more regions adjacent to the rotation center. For $\beta=0^{\circ}$, only a few vortex cores are distributed on the antenna surface. The vortex core appears near the leeward side of the antenna, and its track is continuous due to the rotation. The vortex core in the negative direction of the 


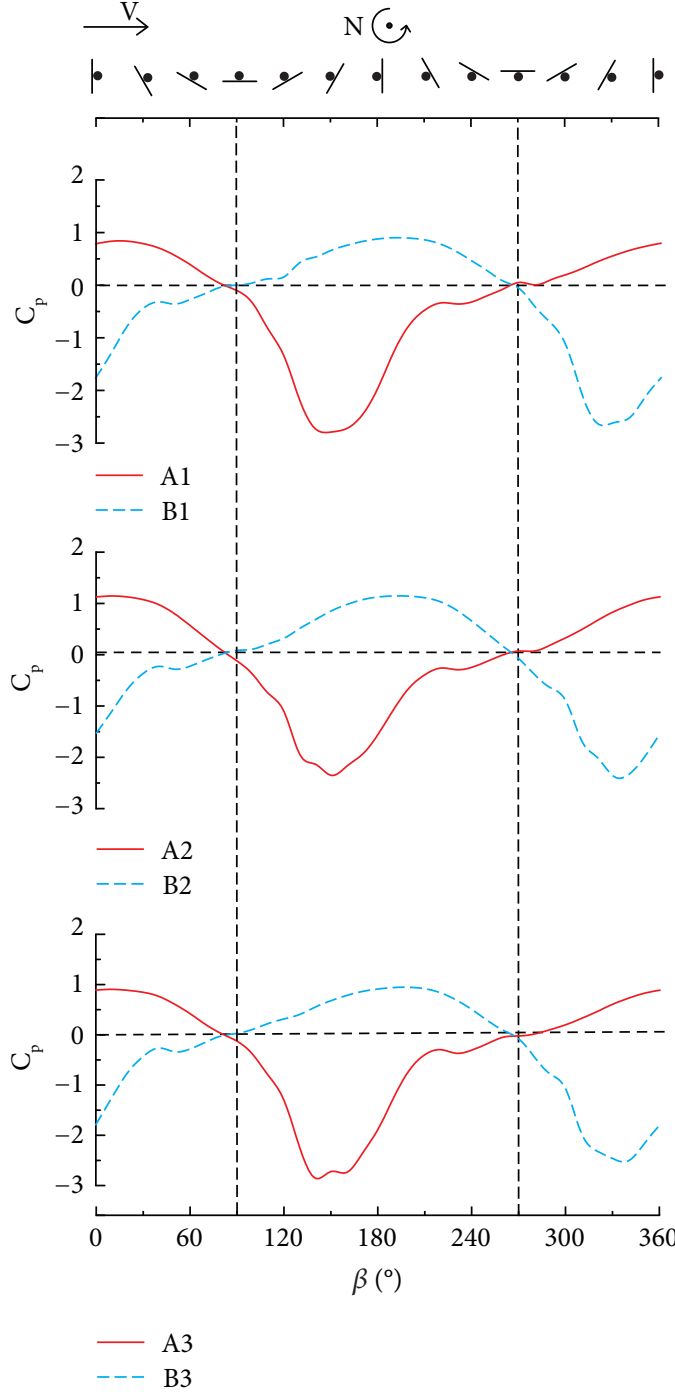

(a)

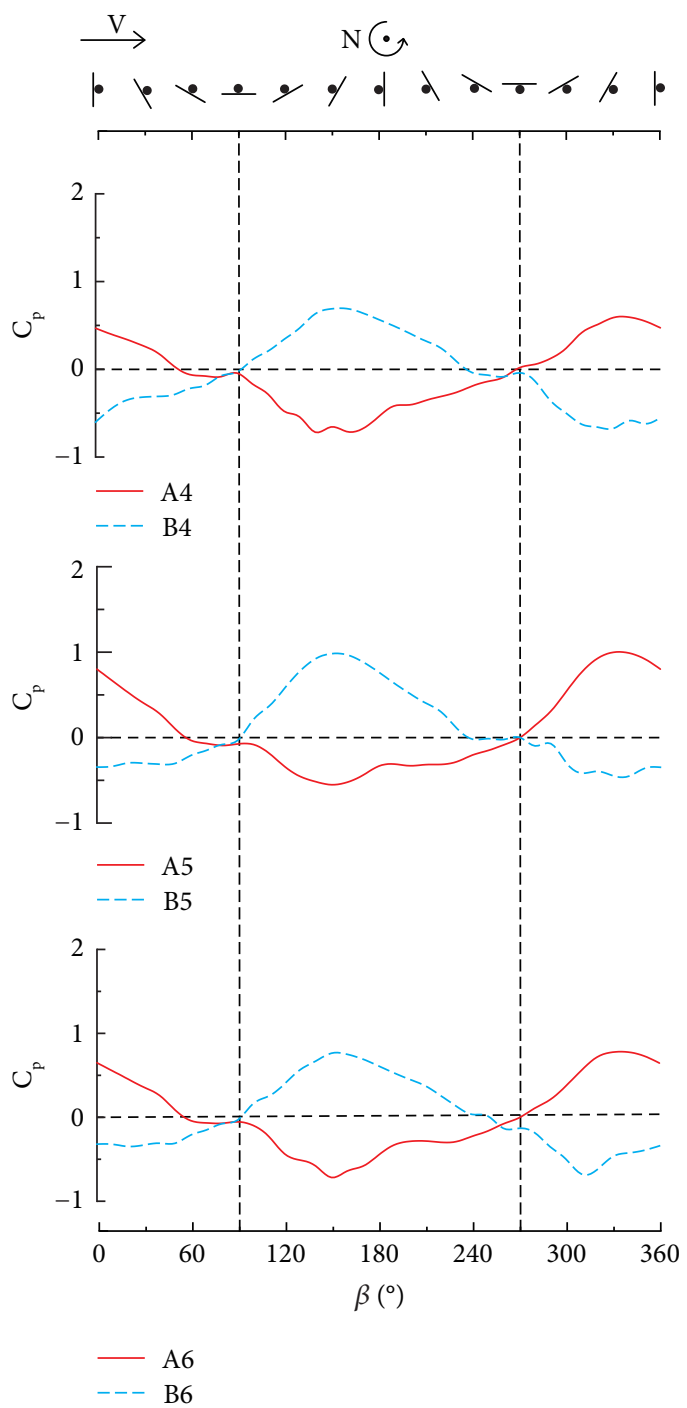

(b)

Figure 12: Pressure coefficients of the surface monitoring point with respect to azimuth angle when the solid plate antenna rotates, $\beta=0-360^{\circ}$, and $K=0.11$. (a) Monitoring points 1,2 , and 3; (b) monitoring points 4,5 , and 6 (A represents the front and B represents the back).

$z$-axis is closer to the antenna, and the antenna produces a positive azimuth moment. At $\beta=30^{\circ}$, the rotation motion increases the dissipation velocity of the vortex core but does not change the position of the vortex core nearby. By $\beta=60^{\circ}$, the vortex cores distributed in the negative direction of the $z$ axis are significantly reduced, and several of them are distributed in the positive direction of the $z$-axis; at this point, the antenna produces a reverse azimuth moment. For $\beta=90^{\circ}$, the continuous vortex core track near the antenna is broken by the rotation motion and the distance between the vortex core and the antenna increases. Combined with Figures 8(d) and 12(b), the aerodynamic coefficient of the antenna is related to the distribution of the vortex core near the antenna, while for the solid plate antenna, the antenna moment is mainly caused by the low pressure formed by the shedding vortex on the leeward side. A similar conclusion has been obtained by Jin and $\mathrm{Xu}$ [25]. In summary, there is some obvious consistency between the antenna surface pressure distribution, the vortex core track distribution, and the antenna aerodynamic coefficient with respect to the azimuth angle.

\subsection{Effects of Rotation Motion on Aerodynamic Characteristics} of Porous Plate Antenna. Designs that increase the porosity rate of the radar antenna can reduce the weight of the servo system and decrease the wind loading on the antenna, thus reducing both design costs and energy consumption. The aerodynamic characteristics of the porous plate antenna at rest and azimuthal rotation are studied, and the effects of azimuthal rotation on its aerodynamic characteristics are analyzed.

4.2.1. Aerodynamic Coefficient of Porous Plate Antenna at Rest. Figure 16 shows the variation of the aerodynamic coefficients with respect to the azimuth angle when the 


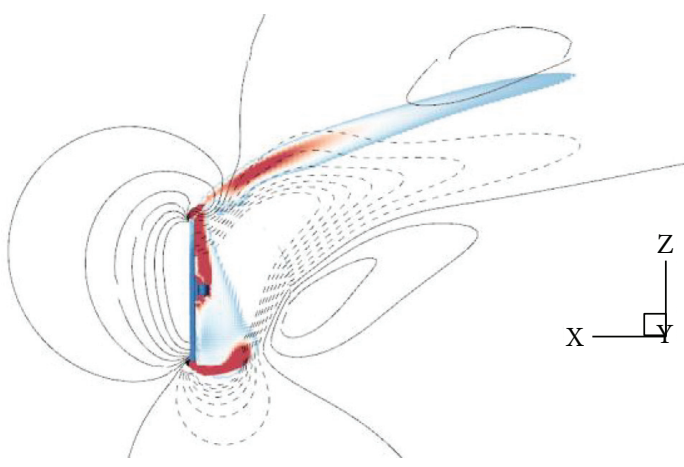

(a)

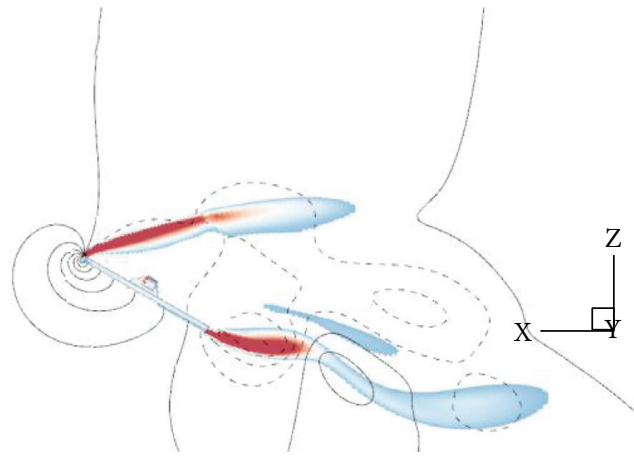

(c)

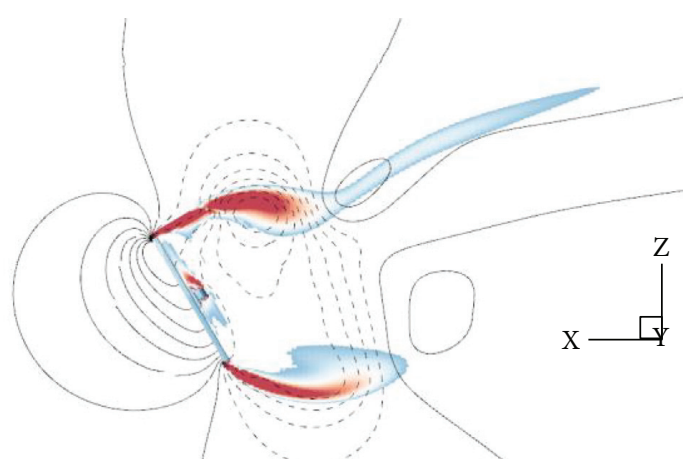

(b)

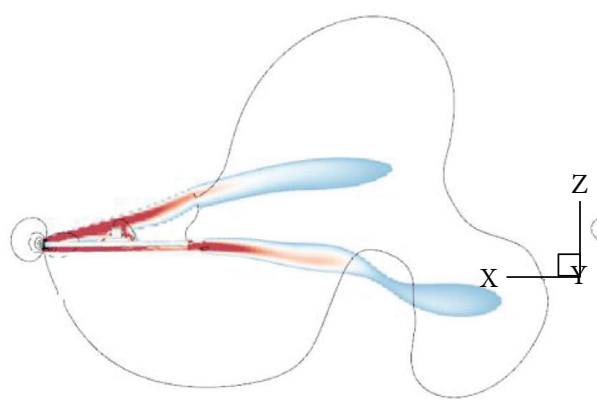

(d)

FIGURE 13: Vorticity nephogram and pressure isosurface nephogram (black solid line represents positive pressure value, black dotted line represents negative pressure value, and color cloud images represent vorticity), (a) $\beta=0^{\circ}$, (b) $\beta=30^{\circ}$, (c) $\beta=60^{\circ}$, and (d) $\beta=90^{\circ}$.

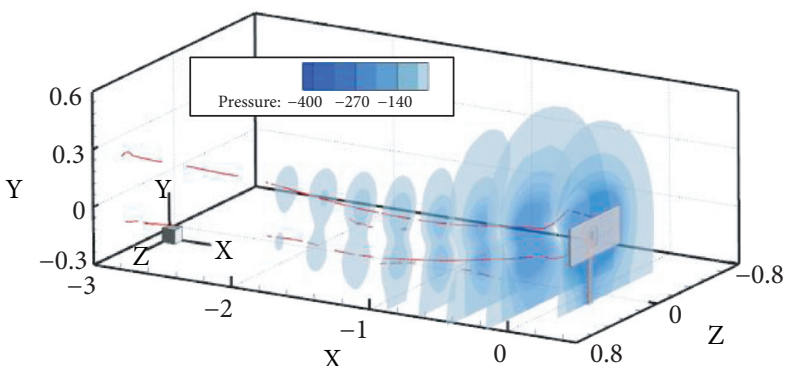

(a)

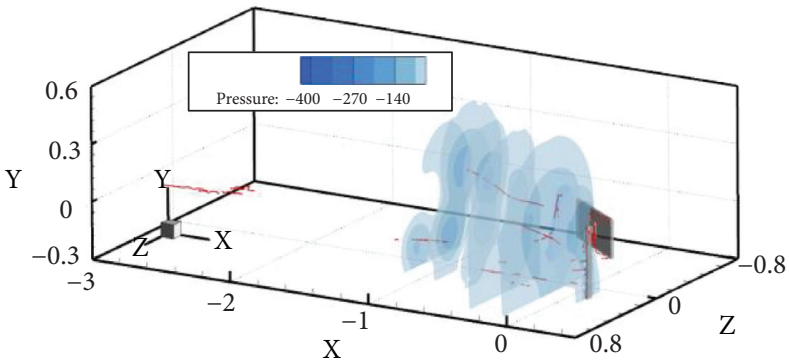

(c)

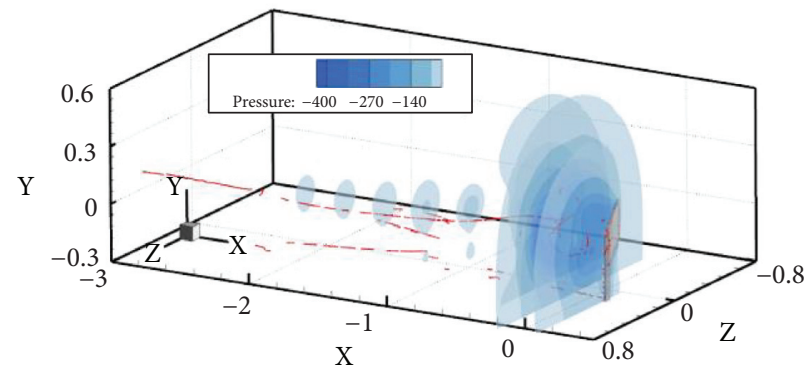

(b)

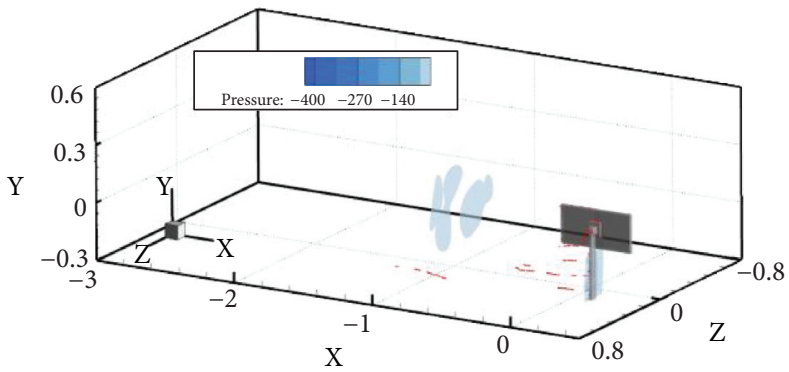

(d)

FIgURE 14: Vortex core track and static pressure YOZ multisection pressure nephogram distribution with azimuth angle. (a) $\beta=0^{\circ}$, (b) $\beta=30^{\circ}$, (c) $\beta=60^{\circ}$, and (d) $\beta=90^{\circ}$.

antenna is stationary. It can be seen from this figure that the numerical simulation results are almost completely consistent with the wind tunnel test results, indicating that it is feasible to predict the aerodynamic coefficients of a porous plate antenna at rest using numerical methods. Thus, the simulation results can be used to analyze the flow field 


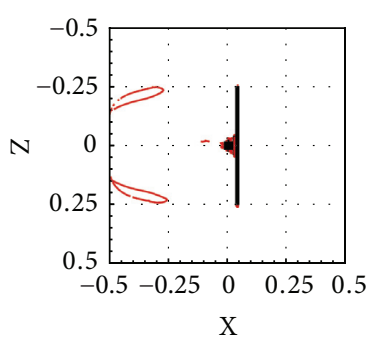

(a)

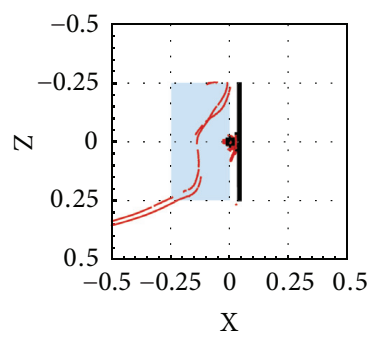

(e)

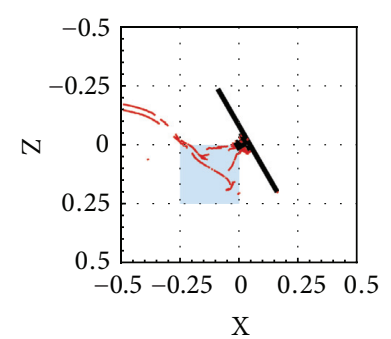

(b)

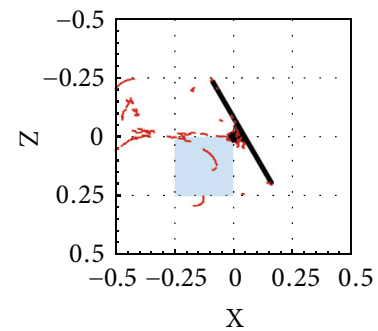

(f)

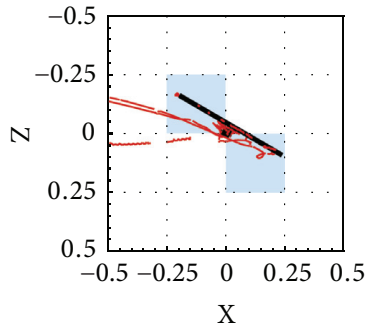

(c)

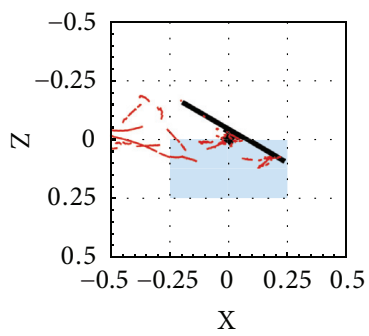

(g)

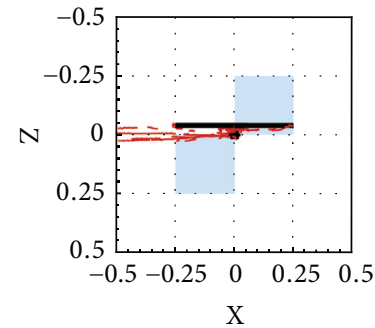

(d)

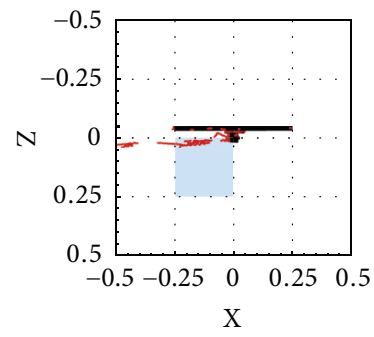

(h)

Figure 15: Vortex cores track at four instantaneous positions for the solid plate antenna. The wind is blowing from right to left. (a)-(d) Stationary and (e)-(h) azimuthal rotation. (a) $\beta=0^{\circ}$, (b) $\beta=30^{\circ}$, (c) $\beta=60^{\circ}$, (d) $\beta=90^{\circ}$, (e) $\beta=0^{\circ}$, (f) $\beta=30^{\circ}$, (g) $\beta=60^{\circ}$, and (h) $\beta=90^{\circ}$.

structure. From a qualitative point of view, the variation in the aerodynamic coefficients with azimuth angle is smooth because the mixing speed of the shedding vortices is fast and uniform, and few large-scale vortices are generated. In addition, the positive peaks of $C_{x}$ and $C_{m z}$ appear at $\beta=0^{\circ}$ and $180^{\circ}$, and the negative peaks appear at $\beta=90^{\circ}$. The positive peaks of $C_{z}$ and $C_{m x}$ appear at $\beta=45^{\circ}$, and the negative peaks appear at $\beta=135^{\circ}$; that is, the peak values of the azimuth moment coefficient occur in opposite directions.

4.2.2. Aerodynamic Coefficient of Porous Plate Antenna during Azimuthal Rotation. Figure 17 shows the variation of the aerodynamic coefficients with respect to azimuth angle as the antenna rotates. It can be seen that the simulated results are slightly different from the test results in terms of peak values, but they are in good agreement with the variation of the azimuth angle. The simulation results can be used to analyze the flow field structure of the antenna. In addition, the test results for the azimuth moment coefficient have an obvious phase lag compared with the simulation results, and the numerical simulation results for $C_{m y}$ at $\beta=215^{\circ}$ reach the maximum negative azimuth moment coefficient ahead of the wind tunnel test results. This is because the faster rotational angular velocity hinders the downstream development of vortices; similar phenomena have been reported by Shields and Mohseni [21].

4.2.3. Comparison of Aerodynamic Coefficients between Stationary and Azimuthal Rotation of Porous Plate Antenna. Figure 18 shows the wind tunnel test results of the aerodynamic coefficients when the antenna is stationary and the azimuthal rotation in the range of $\beta=0-360^{\circ}$. The aerodynamic results of the two states are significantly different in both value size and variation. In addition, when the antenna changes from static to azimuthal rotation, the azimuth moment coefficient and pitching moment coefficient exhibit obvious hysteresis, and the value of the static azimuth moment coefficient precedes the dynamic aerodynamic coefficient. Note that, over one rotation period, the azimuth moment coefficient of antenna rotation is negative at most azimuth angles, although the direction of the moment is the same as the direction of rotation. In contrast, the azimuth moment of antenna rotation is positive at most azimuth angles, and the direction of the moment is the same as that of the rotation.

Table 4 gives the wind tunnel test results for the mean, maximum, and RMS values of the aerodynamic coefficients of the porous plate antenna. In one rotation period, the mean, maximum, and RMS values of the aerodynamic coefficients of the porous plate antenna rotation are enhanced compared with the antenna stationary. Quantitatively, | $C_{x \_}$mean $|,| C_{m y \_}$mean $\mid$, and $\mid C_{m z}$ mean $\mid$ increase by $17.6 \%$, $300 \%$, and $14.8 \%$, respectively; $\left|C_{x}\right| \_\max ,\left|C_{m y}\right| \_\max$, and $\mid$ $C_{m z} \mid \_m a x$ increase by $30.6 \%, 71.4 \%$, and $40.9 \%$, respectively; $C_{x \_} \mathrm{rms}, C_{m y \_} \_\mathrm{rms}$, and $C_{m z}$ rms increase by $22.9 \%, 50 \%$, and $20 \%$, respectively. The results show that the aerodynamic coefficients $C_{x}$ and $C_{m z}$ have negative peaks at $\beta=90^{\circ}$ and positive peaks at $\beta=180^{\circ}, C_{z}$ and $C_{m x}$ have positive peaks at $\beta=45^{\circ}$ and negative peaks at $\beta=135^{\circ}$, and the peak values of the azimuth moment coefficients are in opposite directions when the antenna is stationary. The peak azimuth moment coefficient exhibits a lag when the porous plate antenna is rotating.

4.2.4. Extraction of Surface Pressure Coefficient of Porous Plate Antenna. In Figure 19, C and D represent the front and back of the porous plate antenna, respectively. Each 

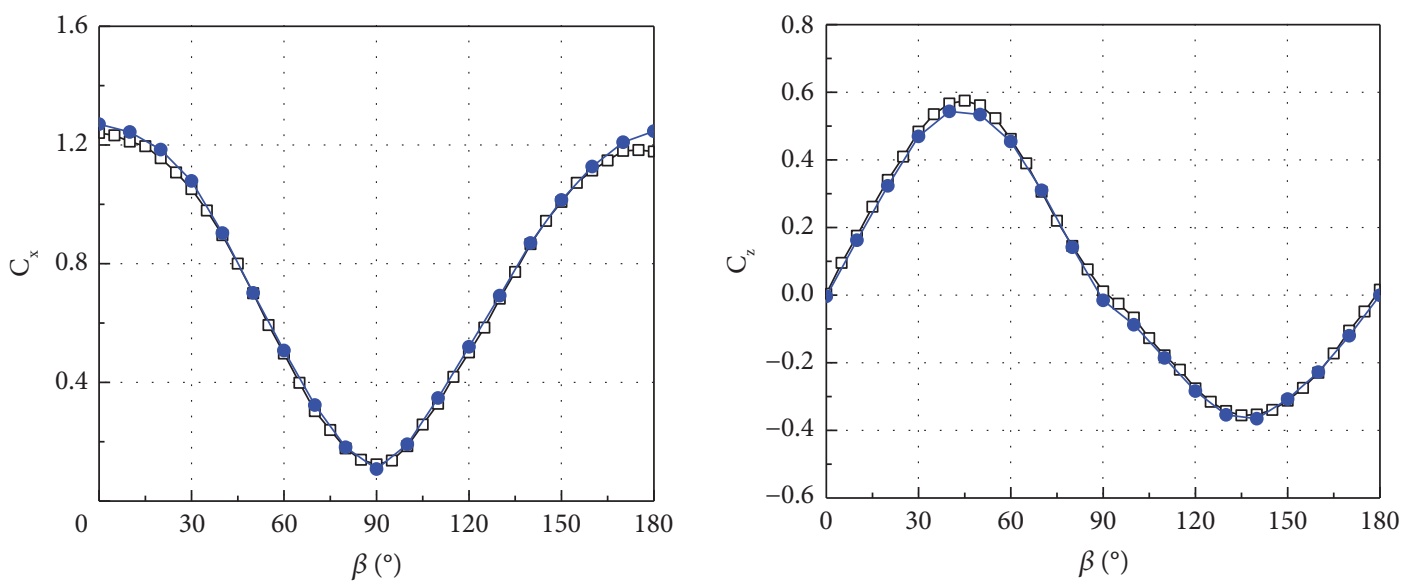

$\rightarrow-$ Exp._stationary

$\rightarrow-$ Exp._stationary

$\rightarrow$ CFD_stationary

$\rightarrow$ CFD_stationary

(a)

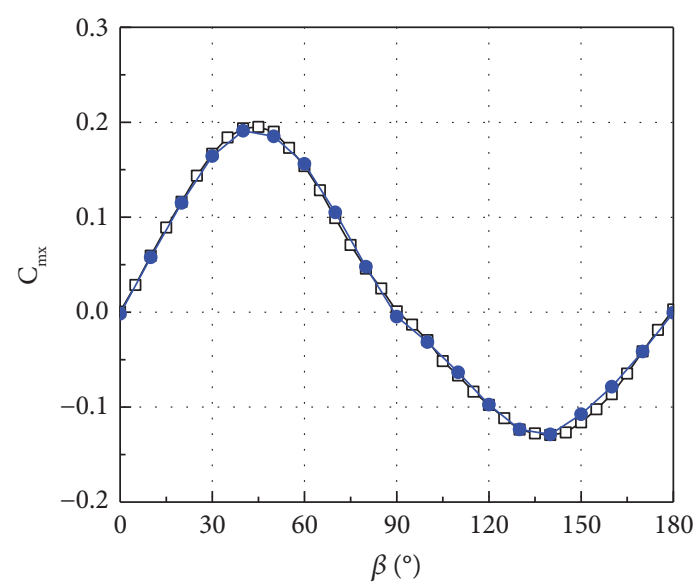

(b)

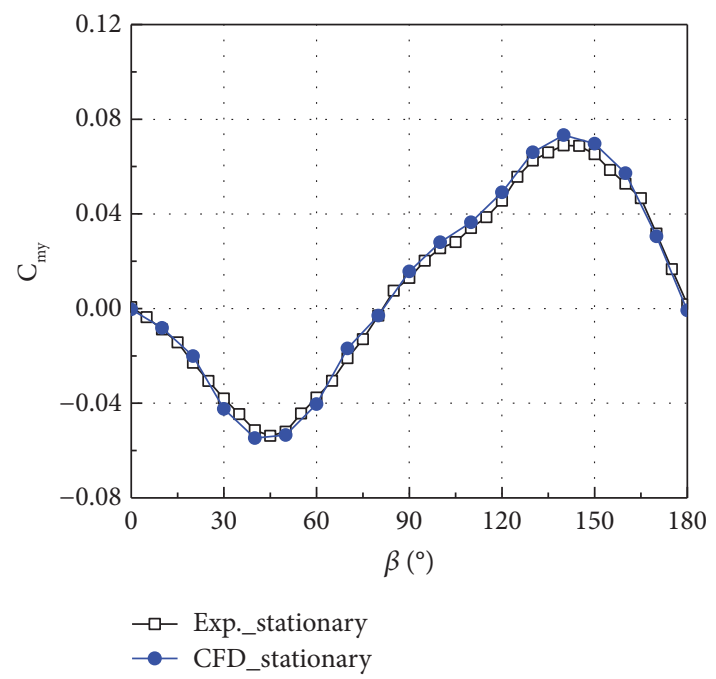

(c)

(d)

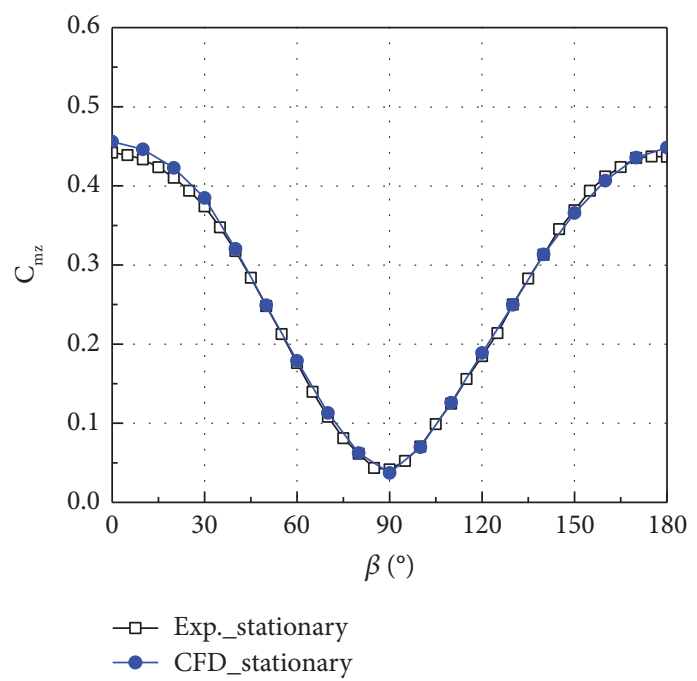

(e)

FIGURE 16: Wind tunnel test results and numerical simulation results of aerodynamic coefficient with respect to the azimuth angle when the antenna is at rest (porous plate, $\beta=0-180^{\circ}$ ). (a) $C_{x}-\beta$, (b) $C_{z}-\beta$, (c) $C_{m x}-\beta$, (d) $C_{m y}-\beta$, and (e) $C_{m z}-\beta$. 


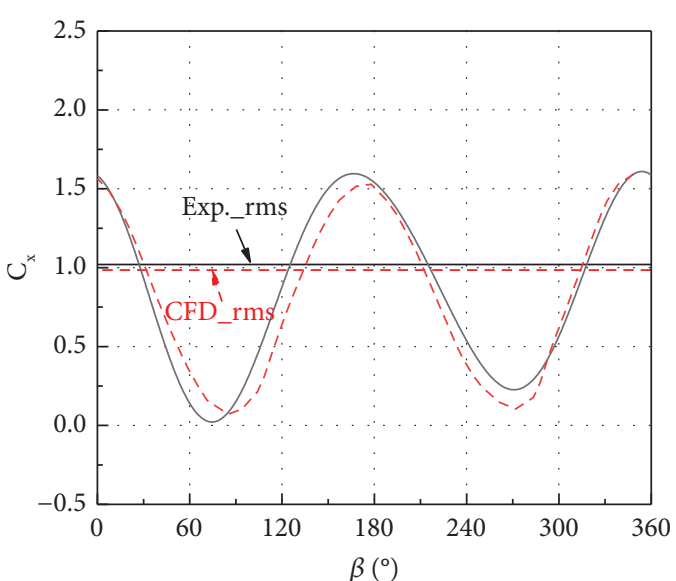

- Exp._rotation - - - CFD_rotation

(a)

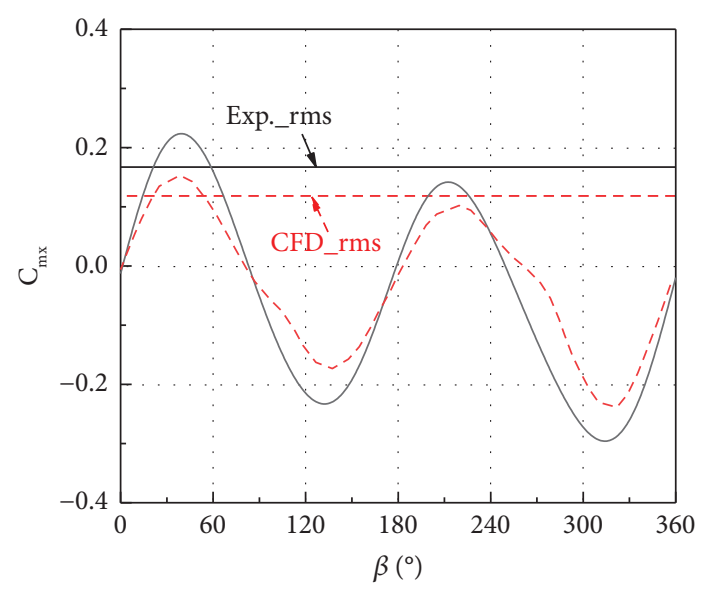

- Exp._rotation

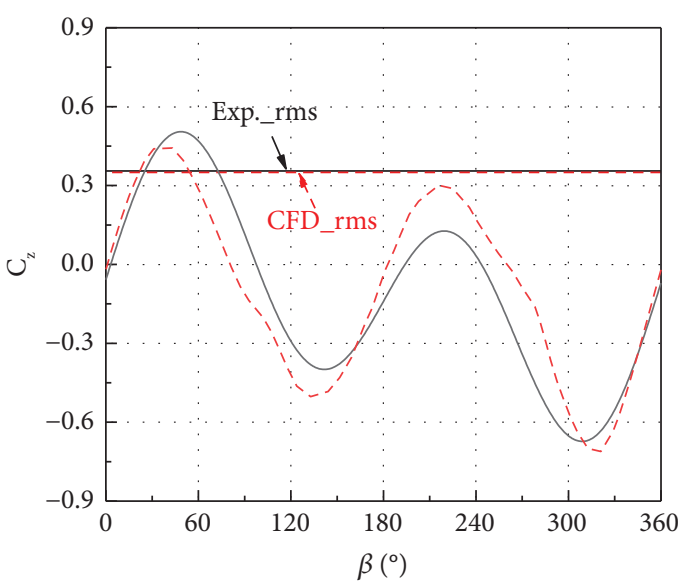

- Exp._rotation

- - - CFD_rotation

(b)

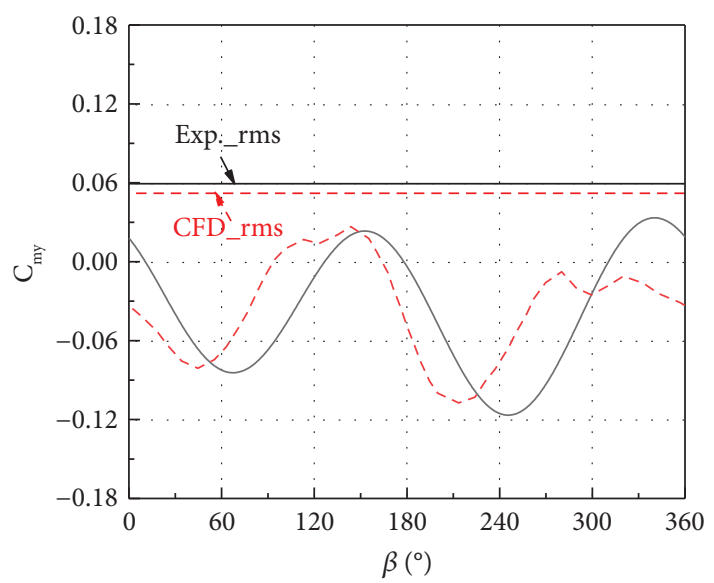

— Exp._rotation

-.- CFD_rotation

(c)

(d)

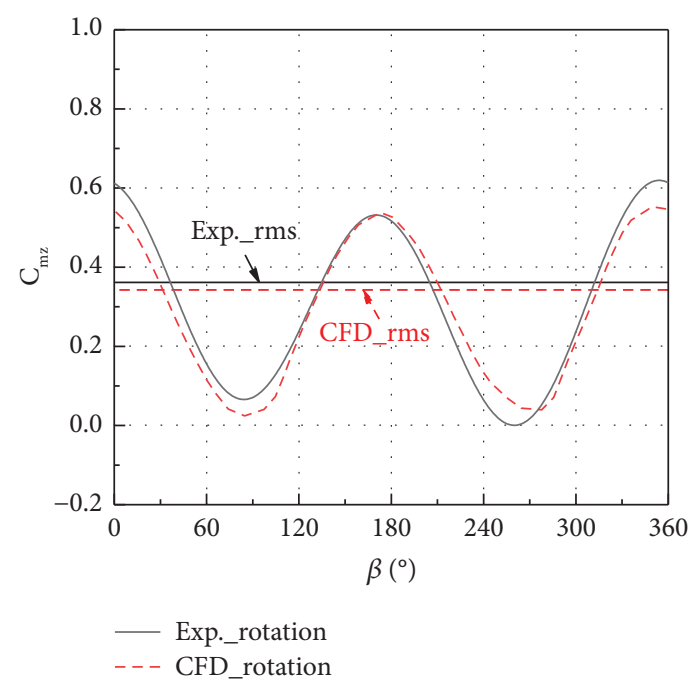

(e)

FIGURE 17: Wind tunnel test results and numerical simulation results of aerodynamic coefficient with respect to azimuth angle when the antenna rotates in azimuth (porous plate, $K=0.34$ ). (a) $C_{x}-\beta$, (b) $C_{z}-\beta$, (c) $C_{m x}-\beta$, (d) $C_{m y}-\beta$, and (e) $C_{m z}-\beta$. 

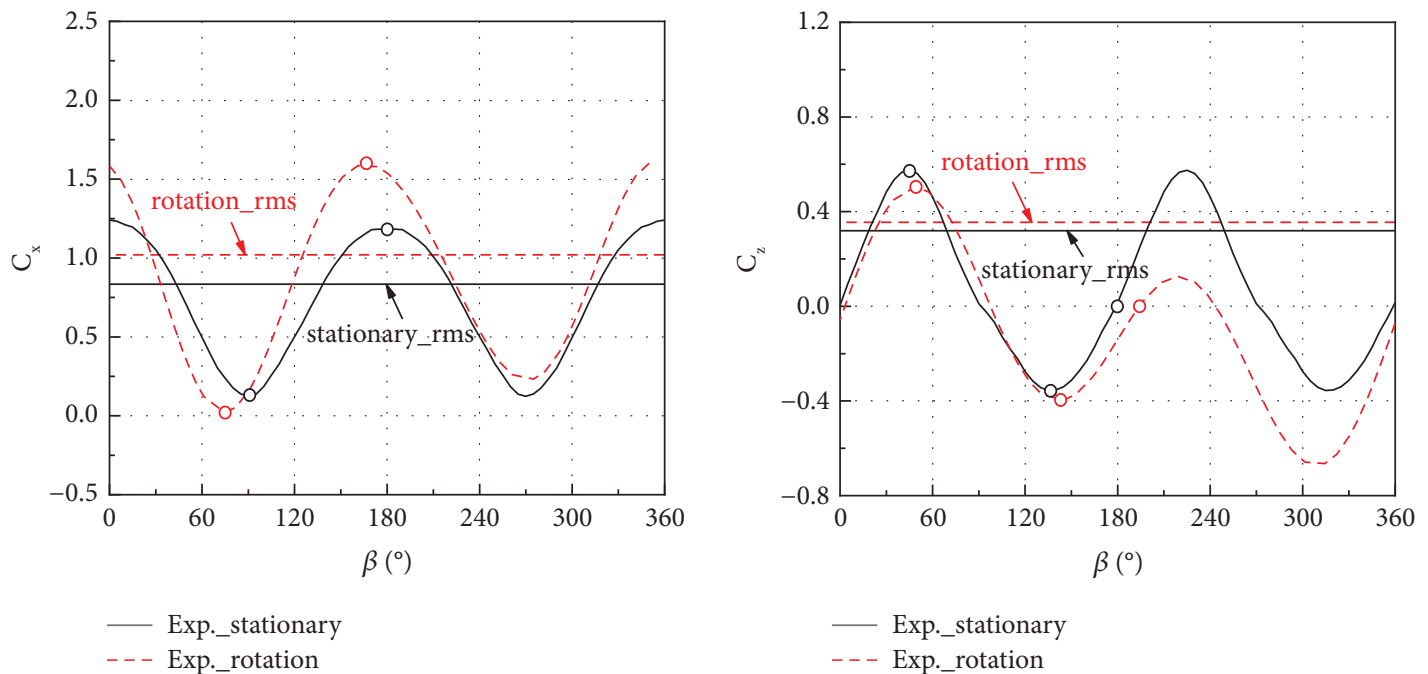

(a)

(b)
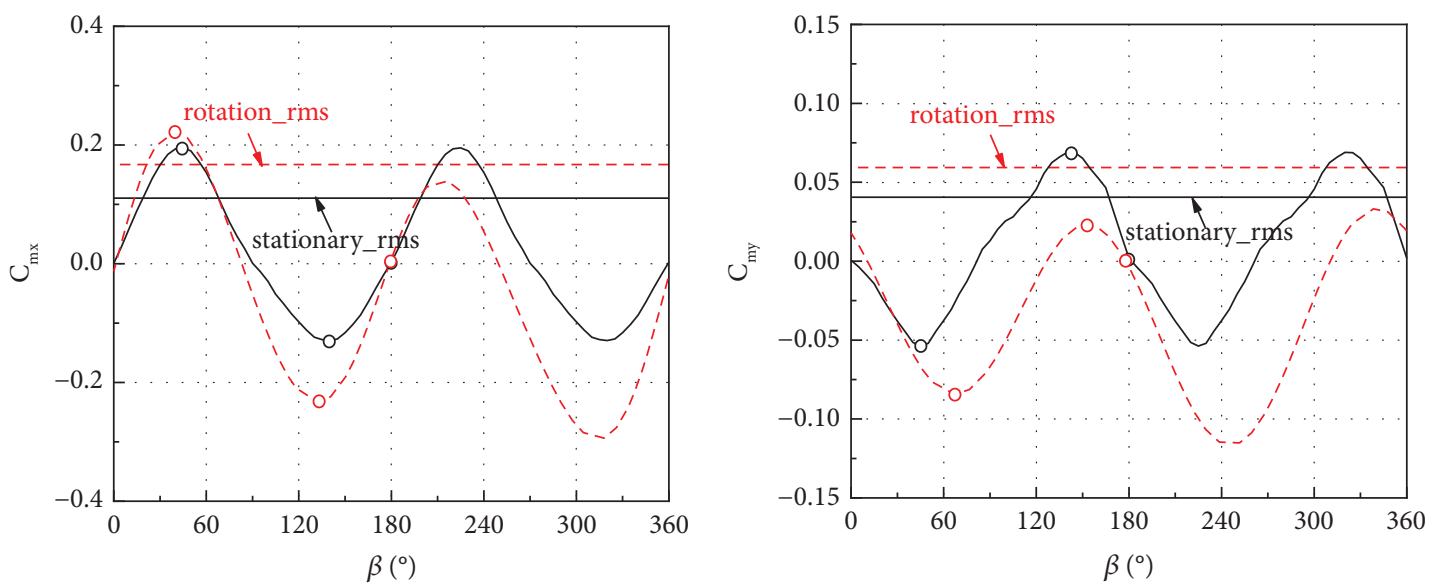

- Exp._stationary
-- Exp._rotation

- Exp._stationary

- - - Exp._rotation

(c)

(d)

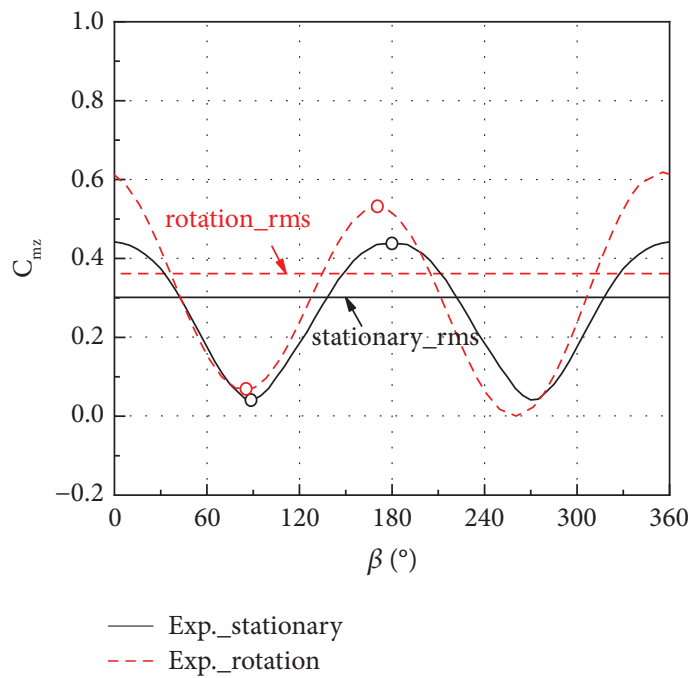

(e)

FIGURE 18: Wind tunnel test results and numerical simulation results of aerodynamic coefficients with respect to the azimuth angle when the antenna is stationary and azimuthal rotation (porous plate). (a) $C_{x}-\beta$, (b) $C_{z}-\beta$, (c) $C_{m x}-\beta$, (d) $C_{m y}-\beta$, and (e) $C_{m z}-\beta$. 
TABLE 4: The mean, maximum, and RMS values of aerodynamic coefficients for the porous plate antenna when the antenna is stationary and azimuthal rotation (the direction is not included in these results).

\begin{tabular}{|c|c|c|c|c|}
\hline \multirow{2}{*}{\multicolumn{2}{|c|}{ Porous plate }} & \multicolumn{3}{|c|}{ Exp. } \\
\hline & & Mean & Maximum & RMS \\
\hline \multirow{5}{*}{ Stationary } & $C_{x}$ & 0.74 & 1.24 & 0.83 \\
\hline & $C_{z}$ & 0.07 & 0.57 & 0.32 \\
\hline & $C_{m x}$ & 0.02 & 0.20 & 0.11 \\
\hline & $C_{m y}$ & 0.01 & 0.07 & 0.04 \\
\hline & $C_{m z}$ & 0.27 & 0.44 & 0.30 \\
\hline \multirow{5}{*}{ Azimuthal rotation } & $C_{x}$ & 0.87 & 1.62 & 1.02 \\
\hline & $C_{z}$ & 0.11 & 0.67 & 0.36 \\
\hline & $C_{m x}$ & 0.05 & 0.30 & 0.17 \\
\hline & $C_{m y}$ & 0.04 & 0.12 & 0.06 \\
\hline & $C_{m z}$ & 0.31 & 0.62 & 0.36 \\
\hline
\end{tabular}

surface has six points near the edges, giving a total of 12 monitoring points. These are used to monitor the changes in the antenna surface pressure coefficient with azimuth angle.

Figure 20 shows the pressure coefficient varies with azimuth angle at each monitoring point on the surface of the porous plate antenna when it is stationary. As shown in the figure, there is no obvious correlation between the pressure coefficients on the left and right sides of the antenna front (back). The fluctuating range of the pressure coefficient at each monitoring point on the antenna surface as the azimuth angle varies is within \pm 1 . This is because the vortices in the flow field around the antenna become fully mixed, and so the pressure distributions on the front and back of the antenna are relatively uniform, which reduces the pressure difference between the front and back of the antenna. In addition, the pressure coefficient values of each monitoring point tend to zero at $\beta=90^{\circ}$ and $270^{\circ}$ on both the front and back of the antenna. The fluctuating value and frequency of the pressure coefficient at monitoring points 1,2 , and 3 change significantly in the range $\beta=90-270^{\circ}$, as shown in Figure 20(a).

Figure 21 shows the variation in the pressure coefficients with azimuth angle at each monitoring point of the porous plate antenna as it rotates. It can be seen that the rotation causes the pressure coefficients at all surface monitoring points to change smoothly with an azimuth angle. The rotation accelerates the dissipation of the vortices generated by the porous plate antenna, and the flow field distribution near the antenna becomes more uniform. Therefore, for pressure monitoring points on the same side of the antenna, the variation in the surface pressure coefficient tends to be consistent with respect to changes in azimuth angle, namely, for points $\mathrm{C} 1, \mathrm{C} 2$, and C3; points C4, C5, and C6; points D1, D2, and D3; and points $\mathrm{D} 4, \mathrm{D} 5$, and D6. In addition, near $\beta=90^{\circ}$ and $270^{\circ}$, the pressure coefficients of the monitoring points on the front and back of the antenna tend to zero, and the pressure coefficient on the back is greater than that on the front.
4.2.5. Extraction of Vortex Structures of Porous Plate Antenna. Figure 22 shows the distribution of vortex core positions in the flow field of the porous plate antenna at $\beta=0^{\circ}, 30^{\circ}, 60^{\circ}$, and $90^{\circ}$. It can be seen that when the antenna is stationary, the vortex cores are evenly distributed around the antenna, the vortex core distribution region is small, and the vortex core track is mostly discontinuous segments. When the antenna rotates, the vortex core track is again discontinuous, but the number and extent of the vortex cores increase significantly.

To analyze the effect of the vortex core positions on the aerodynamic coefficient of the antenna, the region with the highest density of vortex cores around the antenna is roughly illustrated by the blue squares in Figure 22. The vortex core tracks are not continuous. With changes in azimuth, the positions of the vortex core appear more random. The vortex cores near the antenna are mainly distributed in several regions adjacent to the rotation center. When the antenna is stationary at $\beta=0^{\circ}$, there are almost no vortex cores around the antenna, except for those on the antenna surface. The rotation motion enhances the number of vortex cores on the leeward side of the antenna, most of which are in the negative $z$-axis region, causing the antenna to produce a positive azimuth moment. When the antenna rotates to $\beta=30^{\circ}$, the vortex core distribution region does not change significantly. When the antenna rotates to $\beta=60^{\circ}$, the number of vortex cores originally distributed in the negative direction of the $z$-axis decreases, and most of them become distributed in the positive direction of the $z$-axis. At this time, the antenna produces a reverse azimuth moment. By $\beta=90^{\circ}$, the vortex cores are distributed on the leeward side of the antenna, far away from the incoming flow, by the rotation, and the antenna produces a negative azimuth moment. Combined with Figure 18(d), the aerodynamic coefficient of the porous plate antenna appears to be related to the distribution of the nearby vortex cores, which also affects the pressure distribution on the front and back surfaces. 


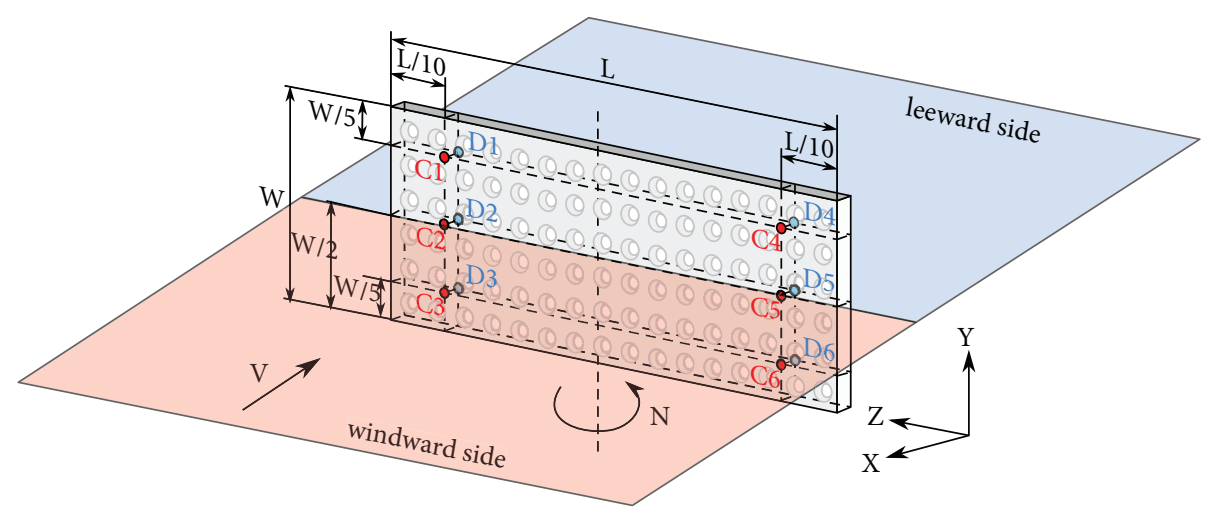

FIGURE 19: Schematic diagram of pressure monitoring points on the surface of the porous plate antenna. Red region represents the windward side of the antenna, blue region represents the leeward side of the antenna, and rotation is counterclockwise.

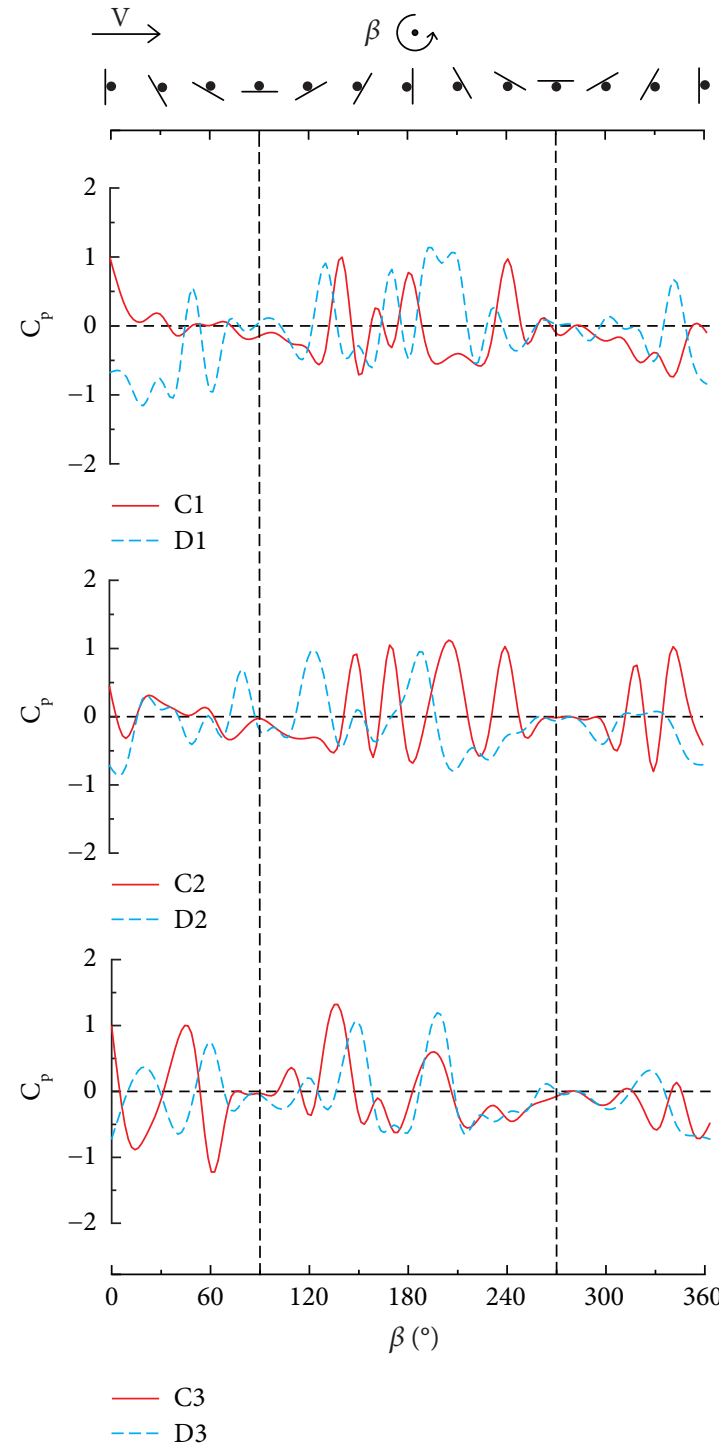

(a)

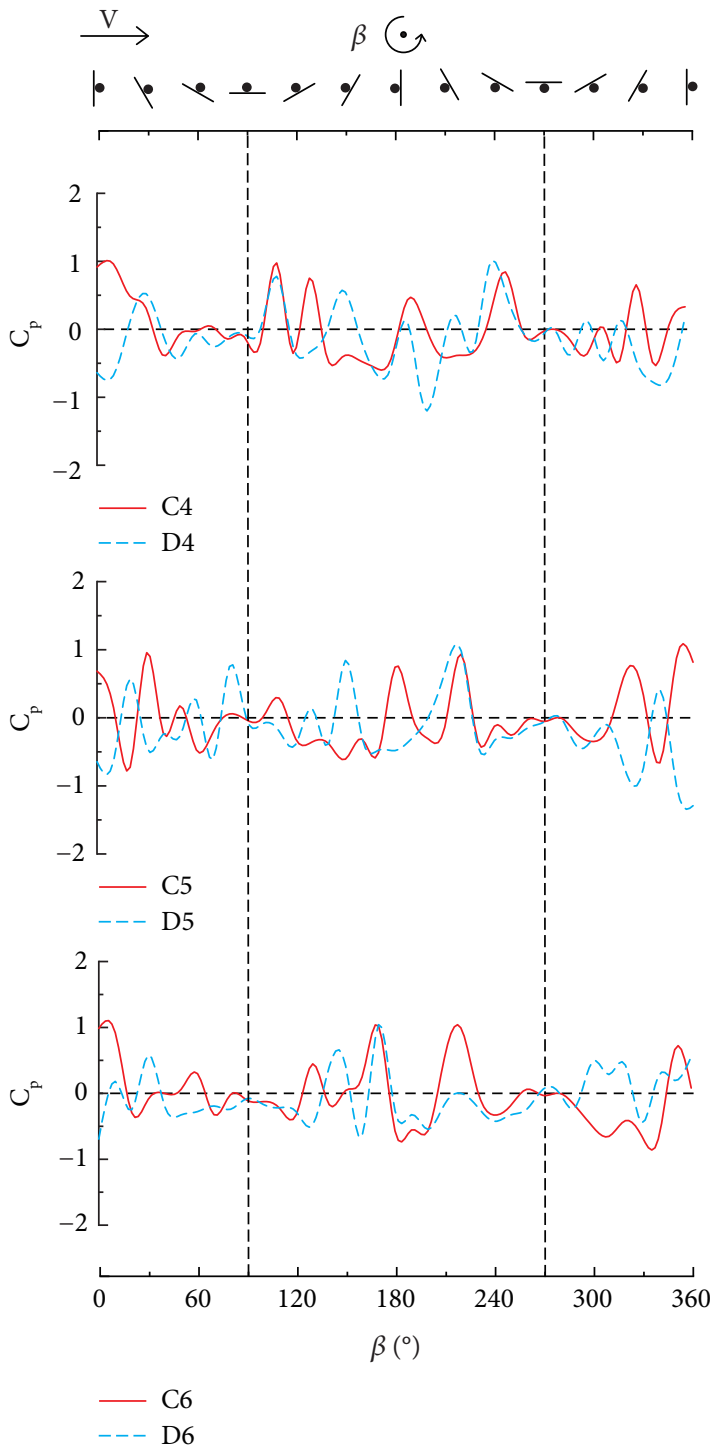

(b)

FIgURe 20: Pressure coefficients of the surface monitoring points with respect to azimuth angle when the porous plate antenna is stationary, $\beta=0-360^{\circ}$, and $\Delta=10^{\circ}$. (a) Monitoring points 1,2 , and 3; (b) monitoring points 4,5 , and 6 (C represents the front of the antenna and $\mathrm{D}$ represents the back of the antenna). 


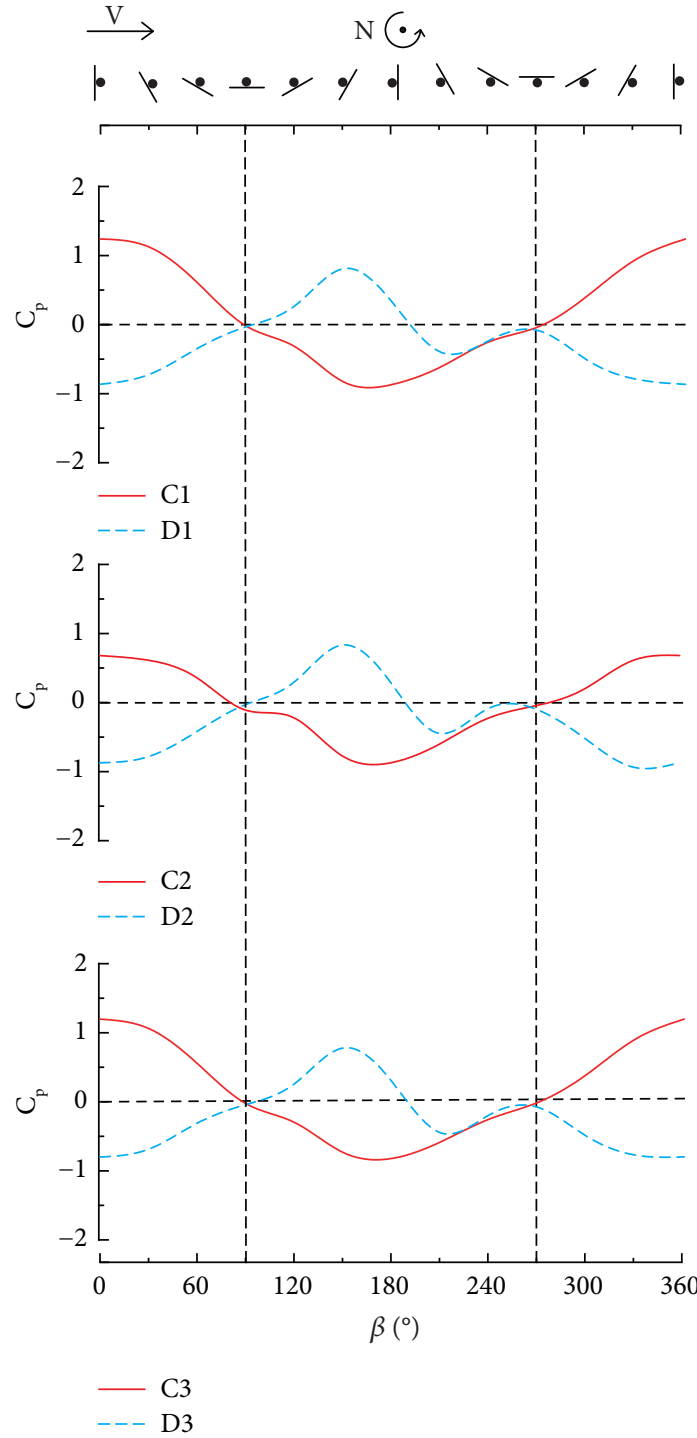

(a)

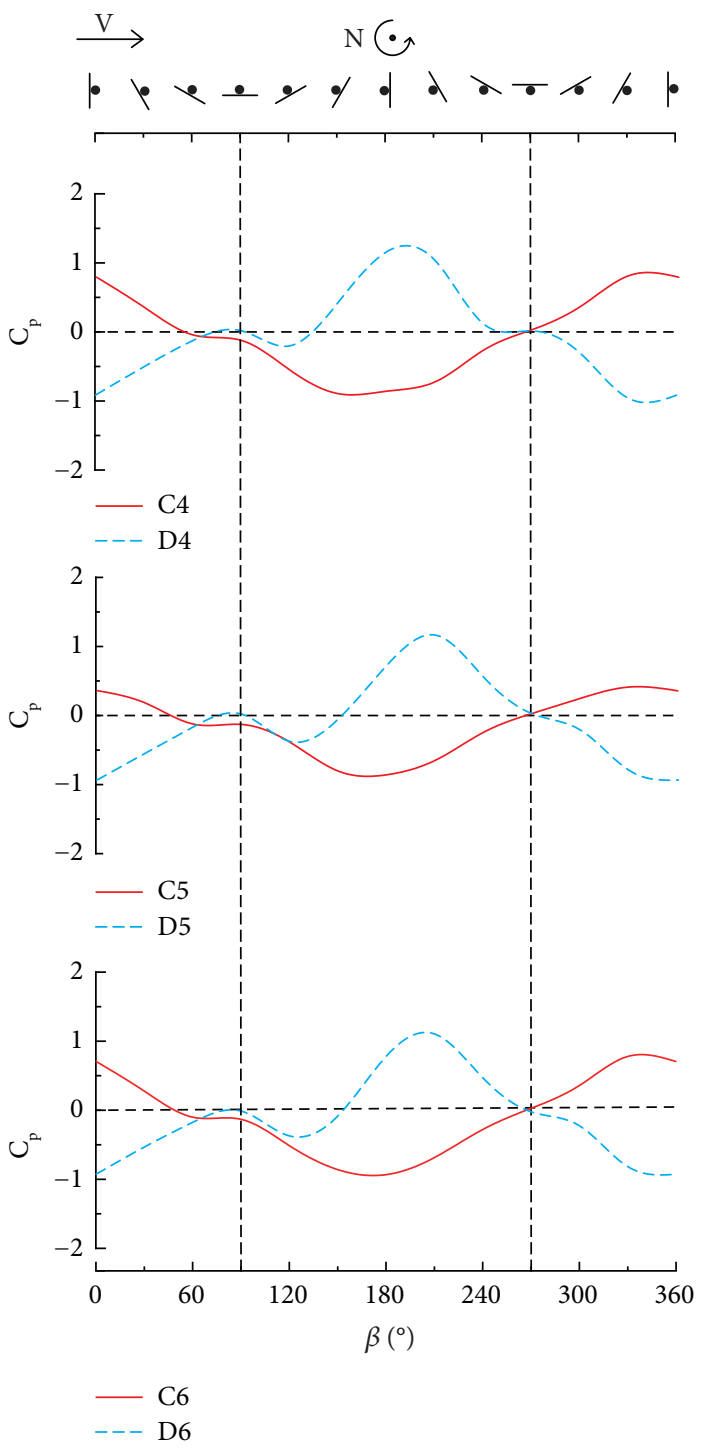

(b)

Figure 21: Pressure coefficient of the surface monitoring points with respect to azimuth angle as the porous plate antenna rotates, $\beta=0-360^{\circ}$, and $K=0.34$. (a) Monitoring points 1,2 , and 3; (b) monitoring points 4,5 , and 6 (C represents the front and D represents the back).

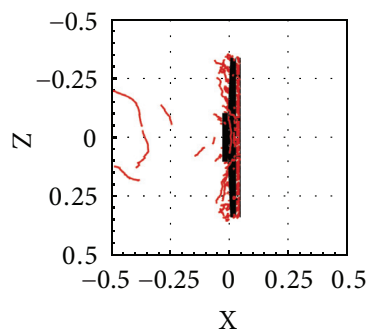

(a)

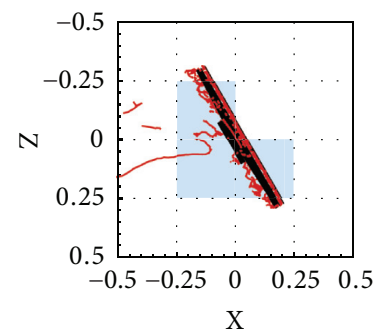

(b)

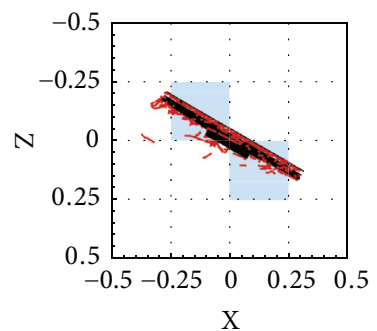

(c)

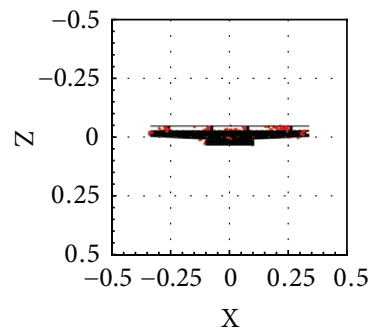

(d)

FIgURE 22: Continued. 


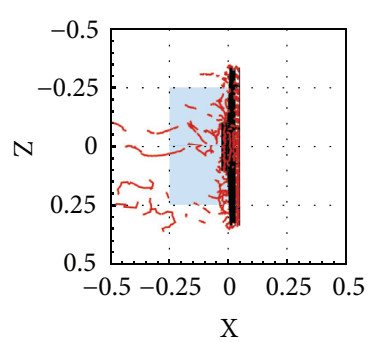

(e)

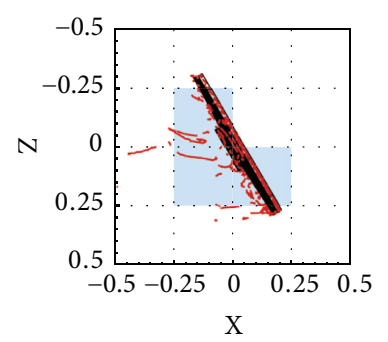

(f)

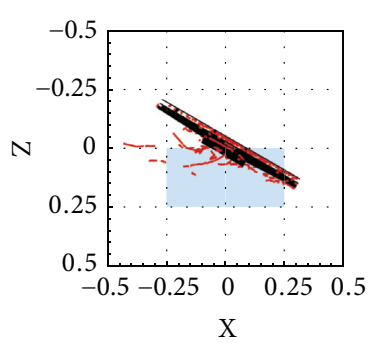

(g)

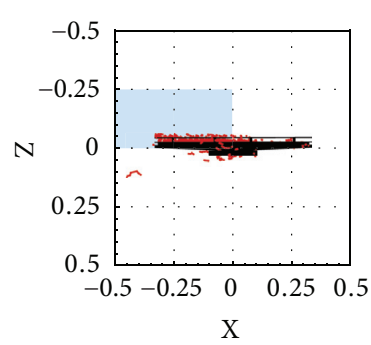

(h)

FIGURE 22: Vortex cores track at four instantaneous positions for the porous plate antenna; the wind is blowing from right to left. (a)-(d) Stationary; (e)-(h) azimuthal rotation. (a) $\beta=0^{\circ}$, (b) $\beta=30^{\circ}$, (c) $\beta=60^{\circ}$, (d) $\beta=90^{\circ}$, (e) $\beta=0^{\circ}$, (f) $\beta=30^{\circ}$, (g) $\beta=60^{\circ}$, and (h) $\beta=90^{\circ}$.

\section{Conclusions}

In this study, the aerodynamic characteristics of a solid plate antenna and a porous plate antenna at rest and during azimuthal rotation have been studied by means of wind tunnel force tests. The variation in the aerodynamic coefficients with respect to the azimuth angle during the antenna is stationary and during azimuthal rotation were then analyzed. Besides, the numerical simulation results corresponding to the working conditions of the wind tunnel test are given. The rationality of the numerical simulation method used in this paper was verified through comparisons with the wind tunnel test results. The pressure and vortex in the flow field are given, the effect of the rotation distribution on the aerodynamic characteristics of the antenna is revealed, and the simulation results provide a reference for analyzing the flow field structure of radar antennas.

A comparison of the wind tunnel test data for the aerodynamic coefficients over one rotation period showed that significant differences occur in the aerodynamic characteristics of the antenna relative to the azimuth angle when the antenna is stationary and when the antenna azimuth rotates. The mean, maximum, and RMS values of the aerodynamic coefficients were found to increase to different extents. The mean, maximum, and RMS values of the drag coefficient, azimuth moment coefficient, and pitching moment coefficient all increase by more than $10 \%$ when the antenna is a solid plate; when the antenna is a porous plate, they all increase by more than $14.5 \%$.

The numerical simulation results show that the aerodynamic characteristics of the antenna are highly correlated with the relative position of the vortex cores in the flow field, and the surface pressure of the antenna decreases as the distance to the vortex cores decreases. For the solid plate antenna, the vortex cores in the flow field around the antenna mainly affect the magnitude and distribution of the pressure on the leeward side of the antenna; for the porous plate antenna, the vortex cores in the surrounding flow field have a greater impact on the pressure on the leeward side of the antenna and on the windward side of the antenna. In addition, there are scattered and discontinuous vortex core tracks around the antenna. This track has little effect on the surface pressure of the antenna, so the continuity of the vortex core tracks near the antenna has a high correlation with the aerodynamic characteristics.

\section{Data Availability}

The data used to support the findings of this study are available from the corresponding author upon request.

\section{Conflicts of Interest}

The authors declare that there are no conflicts of interest regarding the publication of this paper.

\section{Acknowledgments}

This work was supported by the Priority Academic Program Development of Jiangsu Higher Education Institutions (PAPD).

\section{References}

[1] J. D. Holmes, Wind Loading of Structuresp. p333, 3rd edition, CRC Press, London, UK, 2015.

[2] G. Lombardi, "Wind-tunnel tests on a model antenna with different fin configurations," Engineering Structures, vol. 11, no. 3, pp. 134-138, 1989.

[3] G. Lombardi, "Wind-tunnel tests on a model antenna rotating in a cross flow," Engineering Structures, vol. 13, no. 4, pp. 345-350, 1991.

[4] P. Bruger, B. Buchmann, K.-E. Kurrer, and C. Ozimek, "Rotatable shortwave curtain antenna operable at very high wind speeds," IEEE Transactions on Broadcasting, vol. 42, no. 1, pp. 50-54, 1996.

[5] N. L. Fox, "Experimental data on wind-induced vibrations of a paraboloidal reflector antenna model," Report No. NASACR-57422, JPL-CP-5, California Institute of Technology, Pasadena, CA, USA, 1963.

[6] R. B. Blaylock Jr., B. Dayman, and N. L. Fox, "Wind tunnel testing of antenna models*," Annals of the New York Academy of Sciences, vol. 116, no. 1, pp. 239-274, 1964.

[7] M. Kron, "Load distribution on the surface of paraboloidal reflector antennas," Deep Space Network Progress Report, vol. 5, pp. 122-128, 1971.

[8] T. A. Wyatt, "The aerodynamics OF shallow paraboloid antennas," Annals of the New York Academy of Sciences, vol. 116, no. 1, pp. 222-238, 2010.

[9] P. Martín, V. B. Elena, and A. M. Loredo-Souza, "Experimental study of the effects of dish antennas on the wind loading of telecommunication towers," Journal of Wind Engineering and Industrial Aerodynamics, vol. 149, pp. 40-47, 2016. 
[10] P. Sachs, Wind Forces in Engineering, Pergamon Press, Oxford, UK, 2nd edition, 1978.

[11] R. Weerasinghe and T. Bunn, "Wind load assessment of a large radar antenna using measurements and simulation," IOP Conference Series: Earth and Environmental Science, vol. 94, Article ID 012194, 2017.

[12] D. Qiang Du and D. Pingan Du, "Computation of fluctuating wind pressure and wind loads on phased-array antennas," IEEE Antennas and Propagation Magazine, vol. 54, no. 1, pp. 66-75, 2012.

[13] D. Qiang and Y. Yin, "Numerical simulation for wall interference of antennas in wind tunnel test," International Journal of Digital Content Technology and its Applications, vol. 6, pp. 302-309, 2012.

[14] B. Gumusel, C. Camci, and C. Camci, "Aerodynamic drag characteristics and shape design of a radar antenna used for airport ground traffic control," Progress in Computational Fluid Dynamics, An International Journal, vol. 10, no. 1, pp. 32-39, 2010.

[15] C. Camci and B. Gumusel, "Vortex shedding from a ground tracking radar antenna and 3D tip flow characteristics," Progress in Computational Fluid Dynamics, An International Journal, vol. 13, no. 5, pp. 263-269, 2013.

[16] Y. Yu, X. Amandolese, C. Fan, and Y. Liu, "Experimental study and modelling of unsteady aerodynamic forces and moment on flat plate in high amplitude pitch ramp motion," Journal of Fluid Mechanics, vol. 846, pp. 82-120, 2018.

[17] B. Wu, S. Li, S. Cao, Q. Yang, and L. Zhang, "Numerical investigation of the separated and reattaching flow over a 5:1 rectangular cylinder in streamwise sinusoidal flow," Journal of Wind Engineering and Industrial Aerodynamics, vol. 198, Article ID 104120, 2020.

[18] D. M. Hargreaves, B. Kakimpa, and J. S. Owen, "The computational fluid dynamics modelling of the autorotation of square, flat plates," Journal of Fluids and Structures, vol. 46, pp. 111-133, 2014.

[19] F. Gu, J. S. Wang, X. Q. Qiao, and Z. Huang, "Pressure distribution, fluctuating forces and vortex shedding behavior of circular cylinder with rotatable splitter plates," Journal of Fluids and Structures, vol. 28, pp. 263-278, 2012.

[20] AIAA, "Calibration and use of internal strain-gage balances with application to wind tunnel testing (AIAA R-091a-2020)," 2020.

[21] M. Shields and K. Mohseni, "Static and dynamic lateral loads for a low aspect ratio wing at low Reynolds numbers," in Proceedings of the 30th AIAA Applied Aerodynamics Conference, pp. 25-28, New Orleans, LA, USA, June 2012.

[22] F. Gustafsson, "Determining the initial states in forwardbackward filtering," IEEE Transactions on Signal Processing, vol. 44, no. 4, pp. 988-992, 1996.

[23] F. R. Menter, "Two-equation eddy-viscosity turbulence models for engineering applications," AIAA Journal, vol. 32, no. 8, pp. 1598-1605, 1994.

[24] S. Muggiasca, F. Ripamonti, and D. Rocchi, "Numerical and experimental investigation on a maritime radar scanner," in Proceedings of the Fluid Structure Interaction, pp. 313-322, Crete, Greece, January 2009.

[25] C. Jin and K. Xu, "Numerical study of the unsteady aerodynamics of freely falling plates," Communications in Computational Physics, vol. 3, pp. 834-851, 2008.

[26] D. Sujudi and R. Haimes, "Identification of swirling flow in 3D vector fields," in Proceedings of the 12th Computational Fluid Dynamics Conference, CA, U.S.A., 1996. 